\title{
Adaptive Multiresolution Methods for the Simulation of Waves in Excitable Media
}

\author{
Raimund Bürger · Ricardo Ruiz-Baier · Kai Schneider
}

Received: 22 July 2009 / Revised: 6 January 2010 / Accepted: 9 February 2010 /

Published online: 24 February 2010

(C) Springer Science+Business Media, LLC 2010

\begin{abstract}
We present fully adaptive multiresolution methods for a class of spatially twodimensional reaction-diffusion systems which describe excitable media and often give rise to the formation of spiral waves. A novel model ingredient is a strongly degenerate diffusion term that controls the degree of spatial coherence and serves as a mechanism for obtaining sharper wave fronts. The multiresolution method is formulated on the basis of two alternative reference schemes, namely a classical finite volume method, and Barkley's approach (Barkley in Phys. D 49:61-70, 1991), which consists in separating the computation of the nonlinear reaction terms from that of the piecewise linear diffusion. The proposed methods are enhanced with local time stepping to attain local adaptivity both in space and time. The computational efficiency and the numerical precision of our methods are assessed. Results illustrate that the fully adaptive methods provide stable approximations and substantial savings in memory storage and CPU time while preserving the accuracy of the discretizations on the corresponding finest uniform grid.
\end{abstract}

Keywords Spiral waves - Adaptive multiresolution scheme · Finite volume approximation · FitzHugh-Nagumo model · Barkley model $\cdot$ Aliev-Panfilov model

\footnotetext{
R. Bürger

CI²MA and Departamento de Ingeniería Matemática, Universidad de Concepción, Casilla 160-C, Concepción, Chile

e-mail: rburger@ing-mat.udec.cl

R. Ruiz-Baier $(\bowtie)$

Modelling and Scientific Computing, CMCS-MATHICSE-SB, École Polytechnique Fédérale de Lausanne, EPFL, Station 8, 1015 Lausanne, Switzerland

e-mail: ricardo.ruiz@epfl.ch

K. Schneider

Centre de Mathématiques et d'Informatique, Université de Provence, 39 rue Joliot-Curie, 13453 Marseille cedex 13, France

e-mail: kschneid@cmi.univ-mrs.fr
} 


\section{Introduction}

\subsection{Scope}

Nonlinear reaction-diffusion systems are widely used models of excitable chemical and biological media that in many cases exhibit rich spatio-temporal multiscale dynamics. It is well known that even when the medium is homogeneous, patterns with nontrivial spatial structure (pulses, fronts, spiral waves and others) can emerge. We are particularly interested in spiral waves, which have been observed experimentally in excitable media of different origins [22-25, 29, 56, 60, 61, 66]. In these systems, an impulse over a certain threshold initiates a wave of activity moving across the excitable medium. Spiral waves appear, for example, in the solution of systems describing the interaction of activators and inhibitors such as the well-known Belousov-Zhabotinsky reaction [66] (see also [63, 67]), arrhythmias in cardiac tissue [35], fibrillation in atrial tissue, retinal and cortical neural preparations, chemotaxis by amoebae forming multicellular structures [22, 28, 29, 38, 43, 56, 58, 60], patterns on premixed gaseous flame fronts [33, 42, 48, 50], and two-dimensional arrays of electronic circuits [32,49]. (The list of references is far from being complete.) Among these applications, one of the most studied is the propagation of electrical activity in cardiac tissue. This phenomenon involves the interaction of different ion species across a combination of active and passive ion channels and diffusion of charge through a heterogeneous substrate with dynamically changing conductances $[36,46]$. Several tachycardias are closely related to the formation and break-up of spiral waves [37, 68]. The formation and control of spirals has been studied by numerous methods, including bifurcation analysis [39, 54], numerical simulation of PDEs [2, 17, 47, 62, 68] and stochastic PDEs [53, 65], cellular automata [25], matched asymptotic expansions [39], phase equations, and various applicative phenomenological models [42, 43, 52, 56, 57, 60].

In this work, we consider the following spatially two-dimensional model for the description of waves in excitable media, namely a reaction-diffusion system of the generic form

$$
\partial_{t} u=\Delta A(u)+f(u, v), \quad \partial_{t} v=g(u, v),
$$

where $A(u) \geq 0$ is a non-decreasing and Lipschitz continuous diffusion function. The common case of a constant diffusion coefficient is covered by

$$
A(u)=D u, \quad D>0,
$$

but alternatively, we also allow $A(u)$ to vanish on $u$-intervals of positive length, and will utilize in some of the numerical examples the following expression as a prototype model for this situation:

$$
A(u)= \begin{cases}0 & \text { for } u \leq u^{*}, \\ D \cdot\left(u-u^{*}\right) & \text { otherwise }, \quad D>0,\end{cases}
$$

where $u^{*}$ is a given constant. Observe that using (3) turns the $u$-equation in (1) into an ordinary differential equation, and therefore spatial coherence is locally lost wherever $A(u)$ is flat, that is, where $u \leq u^{*}$, so the solution value $u^{*}$ is considered as a threshold for the onset of diffusion. The location of the interface $u=u^{*}$ is not known a priori, but is part of the solution. Degenerate parabolic equations in mathematical biology were first motivated by Witelski [64] (see also [6], where a non-linear, pointwise degenerating diffusion coefficient accounts for a volume filling effect). Numerical solutions to reaction-diffusion systems with 
strongly degenerate diffusion exhibit a behaviour that can be surprisingly different from of the same system with a constant diffusion coefficient when parameters are chosen such that the latter case gives rise to Turing-type pattern formation [5].

The unknowns in (1) are the excitation variable $u=u(x, y, t)$ and the recovery variable $v=v(x, y, t)$, which vary on fast and slow time scales, respectively. The functions $f(u, v)$ and $g(u, v)$ express the local reaction kinetics of the species. A standard choice is the FitzHugh-Nagumo kinetics [36]:

$$
f(u, v)=\frac{1}{\rho}(u(1-u)(u+a)-v), \quad g(u, v)=u-b v,
$$

where the constants $a$ and $b$ control the excitability threshold and duration, and the parameter $\rho>0$ determines the time scale of the fast variable $u$. In this work we will concentrate, however, on the modifications of (4) due to Barkley [2] and Aliev and Panfilov [1], respectively. The model by Barkley [2] is given by

$$
f(u, v)=\frac{1}{\rho} u(1-u)\left(u-\frac{v+b}{a}\right), \quad g(u, v)=u-v,
$$

where the constants $a, b$ and $\rho>0$ play similar roles as in (4). In particular, $\rho$ is usually selected very small, such that the time scale of $u$ can be several orders of magnitude faster than that of $v$. In the application to electrical wave propagation in cardiac models, the "fast" variable $u$ corresponds to the membrane potential, and $a$ and $b$ determine the strength of the transmembrane currents. A larger value of $a$ would increase the action potential duration, whereas a larger ratio $b / a$ increases the excitation threshold. The Barkley model (1), (2), (5) possesses similar dynamics as the FitzHugh-Nagumo model (1), (2), (4), but in addition permits to employ the explicitly given fixed point and nullclines of $u$ and $v$ for the implementation of a fast numerical scheme [3] tailored to the algebraic form of (5). It has therefore become a particularly transparent prototype model for excitable media [31], and has been studied extensively (see e.g. [3, 17, 39]).

The model by Aliev and Panfilov [1] was advanced as a two-variable model for propagation in cardiac tissue, which consists in (1), (2) along with the following variant of (4):

$$
\begin{aligned}
& f(u, v)=-k u(u-a)(u-1)-u v+I_{\mathrm{ext}}, \\
& g(u, v)=\left(c+\frac{d_{1} v}{d_{2}+u}\right)(-v-k u(u-b-1)),
\end{aligned}
$$

where $a, b, c, d_{1}, d_{2}$ and $k$ are certain dimensionless parameters and $I_{\text {ext }}$ denotes an external stimulus, i.e., $I_{\mathrm{ext}}$ is a given time- or time- and space-dependent function. In comparison with the classical FitzHugh-Nagumo kinetics (4), the Aliev-Panfilov kinetics (6) is known to provide more realistic results in the modelling of the electrical activity in ventricular tissue, for example in terms of shape of the action potential curve [52]. In both models (5) and (6), the parameters are dimensionless (as is time $t$ ) and will be specified in Sect. 4, where numerical examples are presented.

It is the purpose of this paper to introduce two variants of a fully adaptive multiresolution (MR) scheme for the efficient computation of solutions to (1) with non-degenerate or degenerate diffusion, (2) or (3), in combination with the kinetics (5) or (6). One variant is based on a standard finite volume scheme (denoted "Scheme A") which can be applied universally to reaction-diffusion systems of the type (1); in particular, this variant can handle both kinetics (5) or (6). A second variant is based on "Scheme B", which in turn is based 
on Barkley's method [2] (see also [3, 17]) devised for (1), (2), (5). Scheme B is based on avoiding evaluating the Laplacian $\Delta A(u)$ wherever $u$ is close to zero, and employs different implicit discretizations of the reaction kinetics in dependence of the location of the current solution value relative to the nullclines of the reaction terms. This method relies on the fact that $u=0$ is a $u$-nullcline for the Barkley kinetics (5), and can therefore in general not be applied to the FitzHugh-Nagumo kinetics (4). We demonstrate by numerical experiments that MR schemes based on both schemes attain considerable speed-up and data compression while preserving the precision of the underlying scheme. In particular, it turns out that for Scheme B applied to (1), (2), (5), the width of the boundary layer yields a nearly optimal reference tolerance for the thresholding procedure within the MR method. On the other hand, in light of the almost radial structure of spiral wave-type solutions, spatially onedimensional computations are sufficient to identify the optimal threshold parameter prior to two-dimensional simulations, so for the problem at hand the implementation of the fully adaptive MR device produces smaller "overhead" of additional computations than for a general two-dimensional reaction-diffusion system [5].

We emphasize here that while the MR device is defined for, and presented herein (in Sect. 3.1) in a rather general and abstract form, while the MR structure we will actually use in this work is based on Cartesian meshes.

\subsection{Related Work}

The excitation dynamics of the PDE model (1) emerges from its ODE version

$$
\frac{\mathrm{d} u}{\mathrm{~d} t}=f(u, v), \quad \frac{\mathrm{d} v}{\mathrm{~d} t}=g(u, v),
$$

and is similar for the kinetics (4), (5) and (6). The behaviour can be illustrated most easily for the Barkley model (5), since the fixed point and nullclines are explicitly given and simply structured, see Fig. 1. Similar diagrams for this case can also be found in [2, 3, 31, 53]; ours contains, in addition, some trajectories computed numerically by the explicit-implicit first-order in time scheme described in [2].

For the parameter values $D=1, \rho=0.05, a=0.75$ and $0 \leq b<b_{\text {cr }}$ with a critical value $b_{\text {cr }} \approx 0.05$, the Barkley model (1), (2), (5) possesses spiral waves [2]. By varying some of these parameters, the trajectories of the spiral wave tip may undergo bifurcations. The problem of finding the rotation frequency and the shape of spiral waves as a function of the parameters in (1), (2), (5) is usually treated by matched-asymptotic expansions of properly scaled versions of this model, see [39] and the references cited in that paper. In general, however, it is not so clear which factors influence, or even compel, the onset of spiral waves. For example, traveling waves may meet an "obstacle" and they "break" [52]. In the context of applications to cardiac problems, this phenomenon is equivalent to a desynchronization of cells in their developmental path.

Excitable media can be characterized by the phenomenon of scale separation, since the time scale of the excitation variable $u$ is essentially shorter than that of the recovery variable $v$. As a strategy to accurately capture the multiscale patterns produced by the difference between time scales, and to concentrate computational effort on zones of strong variation, we present a fully adaptive MR approach. This approach is based on representing the solution at each time on a sequence of nested dyadic grids, where the coefficients of the representation, the so-called details, become small on fine levels of resolution wherever the solution is smooth. Details that are smaller than a level-dependent threshold value may be 


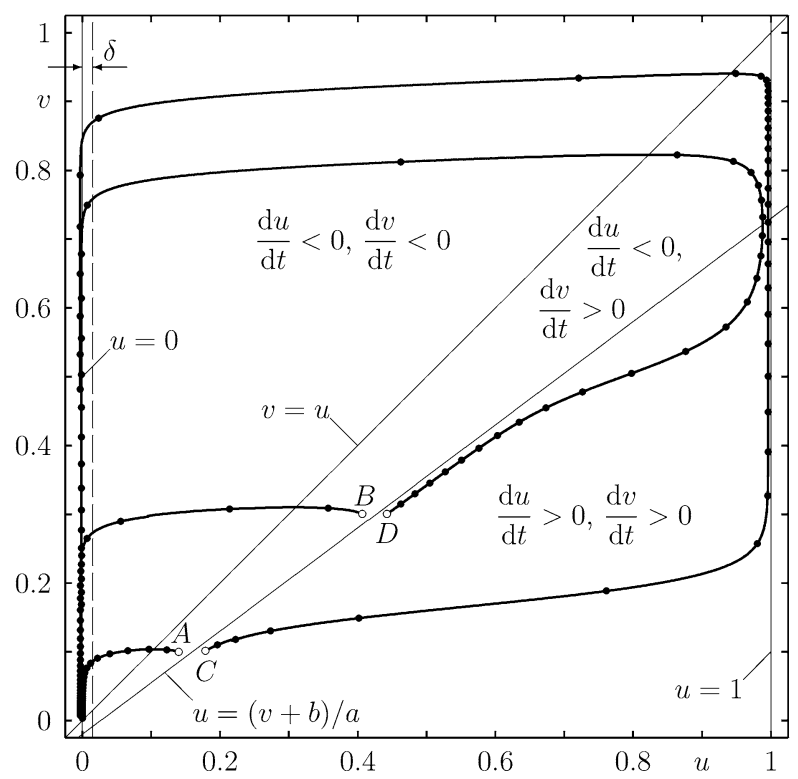

Fig. 1 Schematic phase-space diagram of the ODE system (7) with the Barkley kinetics (5), showing four computed trajectories and the system nullclines: the $v$-nullcline $g(u, v)=0$ consisting of $u=v$, and the $u$-nullcline $f(u, v)=0$ consisting of the three lines $u=0, u=1$ and $u=(v+b) / a$. The parameters are $a=0.75, b=0.02$ and $\rho=0.02$ (as in Examples 1 and 2 of Sect. 4). The origin is the excitable fixed point of the system. Initial conditions close to $(0,0)$ but located to the left of $u=(v+b) / a$ lead to solutions that decay directly into the fixed point, as is shown for the starting points (open circles) $A=(0.14,0.1)$ and $B=(0.41,0.3)$. Initial conditions to the right of $u=(v+b) / a$ cause a large excursion before returning to the fixed point, as is shown for the starting points $C=(0.18,0.1)$ and $D=(0.442455,0.3)$. The fat dots are plotted after every time interval of length 0.1 , and illustrate that the dynamics of the excitation variable $u$ is much faster than that of the recovery variable $v$. The location of the $\delta$-boundary layer is indicated (see Sect. 2.2)

discarded, which allows to substantially compress the representation of the numerical solution in each time step, allowing for substantial data and CPU time savings while controlling the error. Multiresolution techniques were first introduced by Harten [26] to improve the performance of schemes for one-dimensional conservation laws. Later on, the original ideas were extended to related problems [7, 11], leading finally to the concept of fully adaptive multiresolution schemes [13, 15, 44, 51]. Overviews on multiresolution methods for conservation laws are given in $[12,44]$.

In this work, our version of the MR method incorporates Barkley's algorithm [2] to speed up the simulations of waves in excitable media, known as "Barkley's trick" (our Scheme B, see Sect. 2.2), and to the authors' knowledge, it is the first application of MR techniques to spiral waves. Other techniques have been proposed by several authors to accurately solve the system (1). For example, stochastic versions of the model were studied e.g. by Shardlow [53], who uses numerical methods based on Barkley's original scheme and on spectral methods; and the FitzHugh-Nagumo model has been studied also using pseudospectral methods [47]. A nonlinear scaling of $u$ only, which eventually permits an efficient discretization using simple data structures, is proposed in [62], and linear finite element methods combined with a family of partitioning methods is used in [27] to solve related systems. 


\subsection{Initial and Boundary Conditions}

We consider (1) for $(x, y, t) \in Q_{T}:=\Omega \times[0, T]$, where $\Omega \subset \mathbb{R}^{2}$ is an open, bounded, connected polygonal domain with boundary $\partial \Omega$, along with zero-flux boundary conditions

$$
\nabla A(u) \cdot n=0 \quad \text { on } \partial \Omega \times(0, T),
$$

where $n$ is the outer normal vector of $\partial \Omega$, and appropriate initial conditions

$$
u(x, y, 0)=u_{0}(x, y), \quad v(x, y, 0)=v_{0}(x, y) \quad \text { in } \Omega .
$$

For the existence and uniqueness of solutions to the initial-boundary value problem (1), (2), (4) or (5), (8), (9) we refer to results for the FitzHugh-Nagumo equations [59]; see also $[10,21]$. Moreover, in a more general analysis [54], time periodic spiral wave solutions are proved to exist for a class of kinetic formulations.

\subsection{Outline}

The remainder of the paper is organized as follows. In Sect. 2 we recall two numerical methods for obtaining approximate solutions of (1) on uniform fine meshes. These methods play the role of reference numerical schemes, i.e. they are numerical methods to approximate the solution of (1) using a space discretization on a uniform mesh. The first method, Scheme A, is a classical finite volume (FV) scheme based on a first-order Euler time discretization, and the second, Scheme B, is based on "Barkley's trick". Next, in Sect. 3 we will be concerned with the main ingredients of the MR procedure, which allows to construct space-time adaptive schemes based on the reference methods of Sect. 2. The numerical results presented in Sect. 4 confirm the efficiency of the proposed methods, and finally some concluding remarks are given in Sect. 5.

\section{Reference Numerical Schemes}

\subsection{A Standard Finite Volume Method (Scheme A)}

An admissible mesh for $\Omega \subset \mathbb{R}^{2}$ is formed by a family $\mathcal{T}$ of control volumes of maximum diameter $h$ and a family of points $\left(x_{K}\right)_{K \in \mathcal{T}}$ with the following properties (see for instance [18, Definition 5.1]). For a given finite volume $K \in \mathcal{T}, x_{K}$ denotes the center of $K$ and $N(K)$ the set of neighbors of $K$ which share a common edge with $K$. Here $\mathcal{E}_{\text {int }}(K)$ is the set of edges of $K$ in the interior of $\mathcal{T}$ and $\mathcal{E}_{\text {ext }}(K)$ the set of edges of $K$ lying on the boundary $\partial \Omega$. We denote by $\mathcal{E}(K)=\mathcal{E}_{\text {int }}(K) \cup \mathcal{E}_{\text {ext }}(K)$ the set of edges of $K$. For all $L \in N(K), d(K, L)$ denotes the distance between $x_{K}$ and $x_{L}$, and we denote by $\sigma=K \mid L(\sigma=K \mid \partial \Omega$, respectively) the interface between $K$ and $L$ (between $K$ and the boundary $\partial \Omega$, respectively). By $\eta_{K, \sigma}$ we denote the unit normal vector to $\sigma=K \mid L\left(\sigma \in \mathcal{E}_{\text {ext }}(K)\right.$, respectively) oriented from $K$ to $L$ (from $K$ to $\partial \Omega$, respectively). Moreover, $|K|$ stands for the two-dimensional measure of $K$ and $|\sigma|$ for the one-dimensional measure of $\sigma \in \mathcal{E}$. The admissible mesh also satisfies that $\overline{x_{K} x_{L}}$ is orthogonal to $\sigma_{K, L}$. Numerical fluxes on all edges $\sigma$ are defined as follows:

$$
\begin{cases}\tau_{\sigma}\left(A\left(u_{L}\right)-A\left(u_{K}\right)\right) & \text { for } \sigma=K \mid L \in \mathcal{E}_{\text {int }}(K), \\ 0 & \text { for } \sigma \in \mathcal{E}_{\text {ext }}(K),\end{cases}
$$

where the transmissibility coefficients $\tau_{\sigma}$ are defined by $\tau_{\sigma}:=|\sigma| /|d(K, L)|$ for $\sigma=K \mid L \in$ 
$\mathcal{E}_{\text {int }}(K)$. To formulate the underlying scheme, we choose an admissible discretization $\mathcal{D}$ of $Q_{T}$, consisting of an admissible mesh of $\Omega$ and a time step size $\Delta t>0$. We set $t^{n}:=n \Delta t$ for $n \in\{0, \ldots, N\}$, where $N$ is the smallest integer such that $N \Delta t \geq T$. Now we define

$$
f_{K}^{n}:=f\left(u_{K}^{n}, v_{K}^{n}\right), \quad g_{K}^{n}:=g\left(u_{K}^{n}, v_{K}^{n}\right) ;
$$

these quantities are numerical approximations of the respective averages

$$
\frac{1}{|K|} \int_{K} h\left(u\left(x, y, t^{n}\right), v\left(x, y, t^{n}\right)\right) \mathrm{d}(x, y), \quad h \in\{f, g\} .
$$

The initial data are approximated by their $L^{2}$ projections in space:

$$
u_{K}^{0}:=\frac{1}{|K|} \int_{K} u_{0}(x, y) \mathrm{d}(x, y), \quad v_{K}^{0}:=\frac{1}{|K|} \int_{K} v_{0}(x, y) \mathrm{d}(x, y) .
$$

To advance the numerical solution from $t^{n}$ to $t^{n+1}=t^{n}+\Delta t$, we use the following finite volume scheme (Scheme A for short): Given $u_{K}^{n}, v_{K}^{n}$ for all $K \in \mathcal{T}$, determine $\left(u_{K}^{n+1}\right)_{K \in \mathcal{T}}$, $\left(v_{K}^{n+1}\right)_{K \in \mathcal{T}}$ from the following discrete version of (1)

$$
\begin{aligned}
& |K| \frac{u_{K}^{n+1}-u_{K}^{n}}{\Delta t}+\sum_{\sigma \in \mathcal{E}(K)} \tau_{\sigma}\left(A\left(u_{L}^{n+1}\right)-A\left(u_{K}^{n+1}\right)\right)=|K| f_{K}^{n}, \\
& \frac{v_{K}^{n+1}-v_{K}^{n}}{\Delta t}=g_{K}^{n} \quad \text { for all } K \in \mathcal{T} .
\end{aligned}
$$

A CFL stability condition for Scheme A is given by

$$
\frac{\Delta t}{h}\left[\left\|\partial_{u} f\right\|_{\infty}+\left\|\partial_{v} f\right\|_{\infty}+\left\|\partial_{u} g\right\|_{\infty}+\left\|\partial_{v} g\right\|_{\infty}+\frac{4 D}{h^{2}}\right] \leq 1 .
$$

Note that if one chooses initial data $u_{0}$ and $v_{0}$ assuming values in $[0,1]$ then numerical solutions of (1) with either kinetics (5) and (6) remain bounded, with $u(x, y, t) \in[0,1]$, due to the particular algebraic forms of $f(u, v)$ and $g(u, v)$ in these cases. This is transparent for (5), and is confirmed by our numerical results for both cases. Consequently, the norms appearing in (13) can be bounded a priori.

The definition of numerical fluxes (10) includes the zero-flux boundary conditions. That the resulting finite volume scheme has a unique solution and that this solution converges to a weak solution of (1), (2) as the discretization parameters tend to zero follows straightforwardly from classical results (see e.g. [18]). A description of the algorithm can be found e.g. in [4].

\subsection{Local Dynamics and Actively Evaluated Diffusion (Scheme B)}

As proposed in [2], for ease of numerical implementation, the computation for (1), (2) or (3) with the kinetics (5) is split into a semi-implicit integration of the reaction kinetics in the absence of diffusion, and an active evaluation of the diffusion term which permits us to evolve the system more efficiently. This approach is strongly based on the assumption that $u \approx 0$ within a layer of a given small width $\delta$ at the left branch of the $u$-nullcline (see [2] and Fig. 1), meaning that the $u$-field is flat in the interior of recovery regions. Such behaviour is met by model (1), (2) or (3), (5), but not by models that involve the FitzHugh- 
Nagumo kinetics (4). Clearly, Scheme B is not applicable in a general situation. We note that $u=0$ is also a nullcline of the Aliev-Panfilov kinetics (6) whenever $I_{\text {ext }}=0$, and therefore "Barkley's trick", i.e., the active evaluation of the Laplacian $\Delta A(u)$, could potentially be applied to (1), (2) or (3), (6). However, we have found that the largest portion of gain of efficiency for Scheme B applied to (1) and (2) or (3) with the Barkley kinetics (5) is due to the semi-implicit discretization of the reaction terms. These terms are considerably more complicated in the Aliev-Panfilov kinetics (6), so that part of Scheme B cannot be applied.

Further savings can be achieved in other regions, if the Laplacian is evaluated "actively" (see details in [2]). This means that the computation of the approximate Laplacian is incorporated into the algorithm for the local dynamics in such a way that unnecessary calculations are avoided at points whose contribution to the Laplacian of $A(u)$ is zero. Moreover, only for $\Delta t / \rho \leq 1$ can explicit Euler time stepping be used for the time integration of the $u$-dynamics; otherwise, two alternative semi-implicit formulas are employed, depending on whether $u_{K}^{n}$ is located to the left or to the right of the excitability threshold $\hat{u}_{K}^{n+1}:=\left(v_{K}^{n}+b\right) / a$. Each formula prevents the time stepping of the kinetics from overshooting the stable branches of the $u$-nullcline even if $\Delta t$ is large in the "fast" region.

For sake of completeness we detail the main ingredients of one time step of this alternative scheme (Scheme B henceforth) for interior cells in the following algorithm. In this algorithm, $\operatorname{Lap}_{K}^{m}$ denotes an approximate value of $\Delta A(u)$ on $K$, and we recall that $\delta$ is a given small parameter. The variables $m \in\{0,1\}$ and $m^{\prime}=1-m$ flag old and new versions of the discrete Laplacian of $A(u)$.

Algorithm 1 (Scheme B)

Initially $\operatorname{Lap}_{K}^{m}=0$, for all $m \in\{0,1\}, K \in \mathcal{T}$.

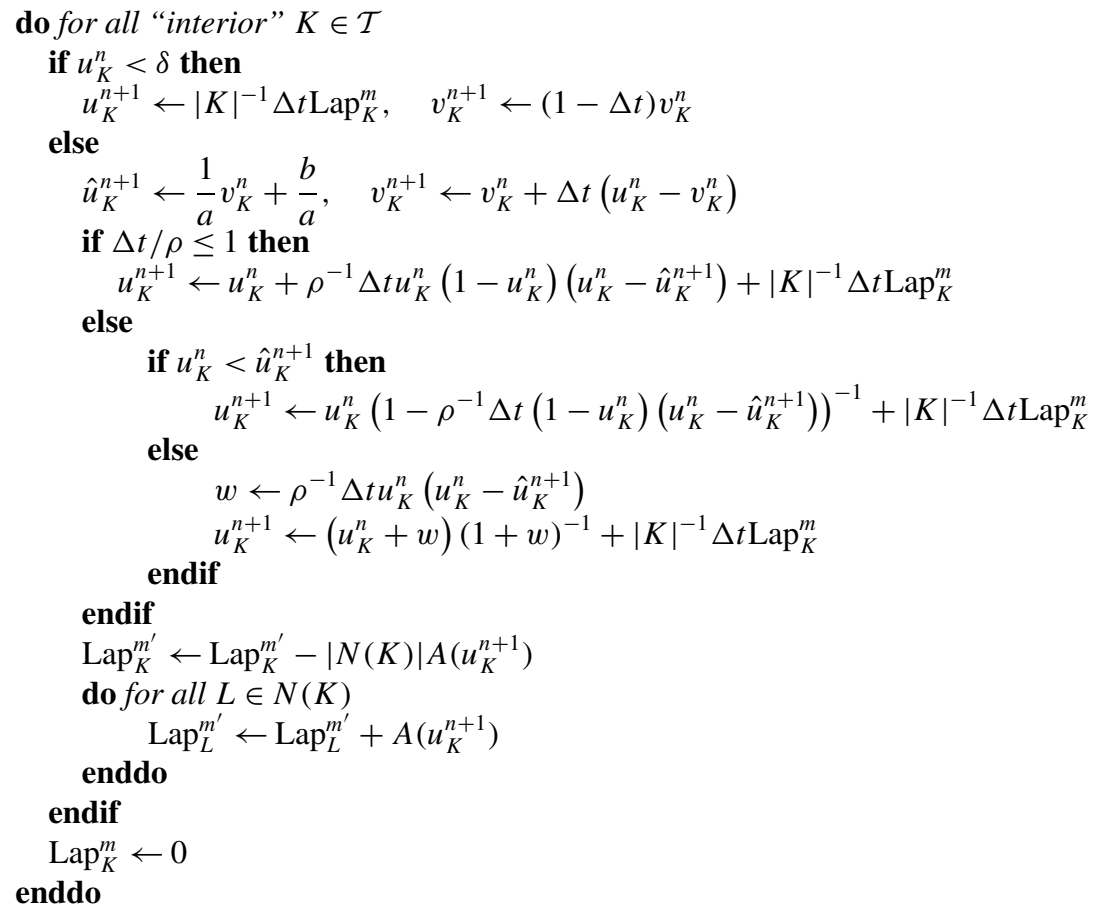

Swap $m$ and $m^{\prime}$. 
For a standard diffusion term (2), the convergence of the numerical solution obtained from Scheme B in the limit $\Delta t, h, \delta \rightarrow 0$ follows from standard arguments [55]. Notice that we update the Lap term in the same way as in Barkley's work [2]. This treatment is possible due to the piecewise linearity of the respective diffusion terms (2) and (3).

\section{Adaptivity: Multiresolution Framework and Local Time Stepping}

In order to equip Schemes A and B with adaptivity, we apply the technique of fully adaptive multiresolution (MR) (see e.g. [5, 13]). This approach basically consists in representing and computing the approximate solution on a dynamically evolving adaptive grid, which is constructed from a sequence of nested grids. On these grids a wavelet basis is defined, from which small coefficients on fine levels of resolution may be discarded (this operation is called thresholding), allowing for substantial data compression. Here we briefly recall some basic aspects of the MR discretization and the data structure which are useful for our study. For a more detailed description we refer to $[8,51]$.

\subsection{Multiresolution Schemes}

For ease of computation, we only consider Cartesian meshes on a rectangular domain, which after a change of variables can be regarded as $\bar{\Omega}=[0,1]^{2}$. Nevertheless, the MR analysis could be carried out for more general meshes, as presented in e.g. [14, 44]. The starting point consists in determining a nested mesh hierarchy $\mathcal{T}_{0} \subset \cdots \subset \mathcal{T}_{H}$, using a partition of $\Omega$. Each grid $\mathcal{T}_{l}$ is formed by the control volumes $K^{l}$ on each level $l, l=0, \ldots, H$, where $l=0$ corresponds to the coarsest and $l=H$ to the finest level. The refinement sets are defined by

$$
\mathcal{M}_{K^{l}}:=\left\{L_{i}^{l+1}\right\}_{i}, \quad \overline{K^{l}}:=\bigcup_{i=1}^{\# \mathcal{M}_{K^{l}}} L_{i}^{l+1}
$$

where $L_{i}^{l+1}$ denotes a control volume at the resolution level $l+1, L_{i}^{l+1} \subset K^{l}$. For $x \in K^{l}$ the scale box function is defined as

$$
\tilde{\varphi}_{K^{l}}(x):=\frac{1}{\left|K^{l}\right|} \chi_{K^{l}}(x)
$$

and therefore the average of any function $u(\cdot, t) \in L^{1}(\Omega)$ in the cell $K^{l}$ can be expressed as the inner product

$$
u_{K^{l}}:=\left\langle u, \tilde{\varphi}_{K^{l}}\right\rangle_{L^{1}(\Omega)}
$$

\subsubsection{Projection and Prediction Operators}

Cell averages and box functions satisfy the two-level relation:

$$
u_{K^{l}}=\sum_{L_{i}^{l+1} \in \mathcal{M}_{K^{l}}} \frac{\left|L_{i}^{l+1}\right|}{\left|K^{l}\right|} u_{L_{i}^{l+1}}, \quad \tilde{\varphi}_{K^{l}}=\sum_{L_{i}^{l+1} \in \mathcal{M}_{K^{l}}} \frac{\left|L_{i}^{l+1}\right|}{\left|K^{l}\right|} \tilde{\varphi}_{L_{i}^{l+1}},
$$

which defines a projection operator needed to move from finer to coarser levels. 
An appealing feature is that we can determine a transformation between the cell averages on level $l=H$ and the cell averages on level $l=0$ plus a series of detail coefficients to be defined later. This transformation is required to be reversible:

$$
\tilde{u}_{K^{l+1}}=\sum_{T \in \bar{S}_{K}^{l}} g_{K, T}^{l} u_{T^{l}}
$$

where $\bar{S}_{K}^{l}$ is the stencil of interpolation or coarsening set, $g_{K, T}^{l}$ are coefficients, and the tilde over $u$ in the left-hand side of (14) denotes a predicted value. Relation (14) defines a prediction operator needed to move from coarser to finer resolution levels. Although this operator is not unique, it is imposed to be consistent with the projection and local. Even with these requirements, there are many operators still available, such as the trivial prediction

$$
\tilde{u}_{L_{i}^{l+1}}=u_{L^{l}} \quad \text { for } i=1, \ldots, \# \mathcal{M}_{K^{l}} .
$$

Typically, however, a polynomial prediction is chosen, which in the particular case of Cartesian meshes is defined by

$$
\tilde{u}_{L_{i}, l+1}=u_{L, l}-Q_{x}-Q_{y}+Q_{x y} \quad \text { for } i=1, \ldots, \# \mathcal{M}_{K^{l}},
$$

where we define

$$
\begin{aligned}
Q_{z} & :=\sum_{n=1}^{s} \tilde{\gamma}_{n}\left(u_{S_{z}, l}-u_{T_{z}, l}\right), \quad z \in\{x, y\}, \\
Q_{x y} & :=\sum_{n=1}^{s} \tilde{\gamma}_{n} \sum_{p=1}^{s} \tilde{\gamma}_{p}\left(u_{S_{x, y}, l}-u_{S_{x,-y}, l}-u_{S_{-x, y}, l}+u_{S_{-x,-y}, l}\right) .
\end{aligned}
$$

Here $S_{ \pm x, \pm y}$ denote the diagonal neighbors of the control volume $S$ and the corresponding coefficients for $s=2$ are (see $[45,51])$

$$
\tilde{\gamma}_{1}=-\frac{22}{128}, \quad \tilde{\gamma}_{2}=\frac{3}{128} .
$$

For $x \in K^{l+1}$ we define the following wavelet function, which in the discrete cell-average multiresolution setting, computes the error between a fine-scale average and its predicted value from coarser scale averages. Therefore the form of this wavelet depends on the choice of the prediction operator. In the case of the choice (15)-(17), one obtains

$$
\tilde{\psi}_{K^{l}, j}=\tilde{\varphi}_{L_{i}^{l+1}}-\sum_{m=-s}^{s} \tilde{\gamma}_{i+m} \tilde{\varphi}_{L_{i+m}^{l}} \quad \text { for } j=1, \ldots, \# \mathcal{M}_{K^{l}} .
$$

The error induced by the prediction operator at the cell $K^{l}$ is defined as the difference between the cell average and the predicted value, i.e.,

$$
d_{K^{l}}:=u_{K^{l}}-\tilde{u}_{K^{l}}
$$

and using the previously introduced wavelet functions, we may also write

$$
d_{K^{l}, j}:=\left\langle u, \tilde{\psi}_{K^{l}, j}\right\rangle \quad \text { for } j=1, \ldots, \# \mathcal{M}_{K^{l}} .
$$


In the case of a multicomponent solution $(u, v)$, on each cell $K^{l}$ they are computed by

$$
d_{K^{l}}=\min \left\{\left|u_{K^{l}}-\tilde{u}_{K^{l}}\right|,\left|v_{K^{l}}-\tilde{v}_{K^{l}}\right|\right\}
$$

for the refinement stages and by

$$
d_{K^{l}}=\max \left\{\left|u_{K^{l}}-\tilde{u}_{K^{l}}\right|,\left|v_{K^{l}}-\tilde{v}_{K^{l}}\right|\right\}
$$

for the coarsening stages of the algorithm.

\subsubsection{Thresholding}

Details are related to the regularity of a given function. Roughly speaking, the more regular a function $u$ is over $K^{l}$, the smaller is the corresponding detail coefficient. This property motivates the so-called thresholding procedure, which basically consists in discarding all control volumes corresponding to details that are smaller in absolute value than a level-dependent tolerance $\varepsilon_{l}$. Choosing $\varepsilon_{l}$ too small or too large will make the multiresolution device inefficient (the compression rate is poor) or deteriorate the quality of the solution due to large thresholding errors, respectively. A reasonable choice of $\varepsilon_{l}$ is based on the consideration that the order of convergence of a given reference scheme should be maintained if this scheme is endowed with multiresolution. Cohen et al. [13] and Roussel et al. [51] advance a calculus, valid for the respective cases of one-dimensional hyperbolic conservation laws [13] and multi-dimensional strictly parabolic equations [51], that leads to a value of the so-called reference tolerance $\varepsilon_{\mathrm{R}}$ provided that among other properties ( $L^{1}$ contractivity of the general time evolution operator, and $L^{\infty}$ stability of the reference numerical scheme) the reference scheme has a known order of convergence in space $(\alpha=1 / 2$ and $\alpha=2$ for the problems considered in [13] and [51], respectively).

The latter constant is at present unknown for finite volume discretizations of degenerate parabolic equations. However, encouraging numerical results in previous papers including $[4,5,8]$ indicate that the methodology of $[13,51]$ can also be successfully applied to degenerate reaction-diffusion systems when $\alpha$ is a convergence rate obtained from a series of numerical experiments. This idea will also be applied in the present situation. To this end, we briefly summarize the basic arguments from [5] adapted to (1). (In fact, the systems investigated in [5] are of the slightly more general type $u_{t}=\Delta A(u)+f(u, v)$, $v_{t}=\Delta B(u)+g(u, v)$.) Assume that $\mathbf{u}=(u, v)$, and that $\overline{\mathbf{u}}_{\mathrm{ex}}^{H}, \overline{\mathbf{u}}_{\mathrm{FV}}^{H}$ and $\overline{\mathbf{u}}_{\mathrm{MR}}^{H}$ denote the cell averages of the exact solution, the cell averages of the numerical solution obtained by the reference finite volume scheme, and the cell averages of the MR numerical solution corresponding to the finest level $H$, respectively. For a given norm $\|\cdot\|$ we can then formally write $\left\|\overline{\mathbf{u}}_{\mathrm{ex}}^{H}-\overline{\mathbf{u}}_{\mathrm{MR}}^{H}\right\| \leq E_{1}+E_{2}$, where $E_{1}:=\left\|\overline{\mathbf{u}}_{\mathrm{ex}}^{H}-\overline{\mathbf{u}}_{\mathrm{FV}}^{H}\right\|$ and $E_{1}:=\left\|\overline{\mathbf{u}}_{\mathrm{FV}}^{H}-\overline{\mathbf{u}}_{\mathrm{MR}}^{H}\right\|$ are the so-called discretization and perturbation errors, respectively. Now suppose we know that there exists a (solution-dependent) constant $C_{1}$ such that $E_{1} \leq C_{1} 2^{-\alpha H}$. Concerning $E_{2}$, our reasoning is based on the analysis by Cohen et al. [13] for scalar conservation laws. They showed that if the discrete time evolution operator is contractive in the chosen norm and details on a level $l$ are deleted when they are smaller than a level-dependent tolerance $\varepsilon_{l}$ given by

$$
\varepsilon_{l}=2^{2(l-H)} \varepsilon_{\mathrm{R}}, \quad l=0, \ldots, H,
$$

where $\varepsilon_{\mathrm{R}}$ is a reference tolerance, then the perturbation error accumulates in time and satisfies $E_{2} \leq C_{2} n \varepsilon_{\mathrm{R}}$, where $C_{2}$ is a constant and $n$ is the number of time steps. At a fixed time 
$T=n \Delta t$ this gives $E_{2} \leq C_{2}(T / \Delta t) \varepsilon_{\mathrm{R}}$. Suppose now that the reference scheme is subject to a CFL condition of the type

$$
\Delta t \leq \Delta x^{2}(a \Delta x+b)^{-1}
$$

(note that (13) can be converted to an inequality of this type), where the constants $a$ and $b$ depend on the coefficients of the equation under consideration, and $\Delta x$ is the meshwidth of the finest grid, i.e. $\Delta x=\tilde{C}(\Omega) 2^{-H}$, where $\tilde{C}(\Omega)$ depends on the dimension and shape of $\Omega$. The largest possible time step can then be expressed in terms of $2^{-H}$ if we consider equality in (19), i.e.,

$$
\Delta t=(\tilde{C}(\Omega))^{2} 2^{-2 H}\left(\tilde{C}(\Omega) 2^{-H} a+b\right)^{-1} .
$$

Now, if $E_{1}$ and $E_{2}$ are supposed to be of the same orders as $\Delta x$ or equivalently, $H$ varies, then we must have a proportionality of the type $\varepsilon_{\mathrm{R}} \propto 2^{-\alpha H} \Delta t$. Replacing $\Delta t$ by the righthand side of (20), which in turn represents the CFL condition (as stated above), we obtain an explicit expression for the reference tolerance provided that the factor of proportionality $C$ in $\varepsilon_{\mathrm{R}}=C 2^{-\alpha H} \Delta t$ is chosen appropriately.

Let us now denote by $\alpha$ the experimental convergence rate of (11)-(12), which by means of standard preliminary computations (see Example 1) we have found to be $\alpha=1.2$. Under the assumptions of $L^{1}$ contractivity of the general time evolution operator and stability of the reference numerical scheme (13), and following the principles outlined above, we then obtain the following expression for $\varepsilon_{\mathrm{R}}$ :

$$
\varepsilon_{\mathrm{R}}=C \frac{2^{-(\alpha+2) H}}{|\Omega|\left(\left\|\partial_{u} f\right\|_{\infty}+\left\|\partial_{v} f\right\|_{\infty}+\left\|\partial_{u} g\right\|_{\infty}+\left\|\partial_{v} g\right\|_{\infty}\right)+D|\Omega|^{3 / 2} 2^{2+H}},
$$

see e.g. [5] for its deduction. The level-dependent tolerances $\varepsilon_{l}$ are then given by (18), which guarantees that the error due to thresholding is of the same order as the discretization error, and therefore the order of the underlying scheme is preserved. The constant $C$ in (21) has to be determined by test calculations on a uniform grid, possibly in one space dimension only, prior to the proper multiresolution simulation. This is documented in detail for Example 4 in Sect. 4.

\subsubsection{Dynamic Graded Tree}

We organize the cell averages and corresponding details at different levels in a dynamic graded tree. The root is the basis of the tree. A parent node has four sons, and the sons of the same parent are called brothers. A node without sons is a leaf. A given node has $s^{\prime}=2$ nearest neighbors in each spatial direction, needed for the computation of the fluxes of leaves; if these neighbors do not exist, we create them as virtual leaves. Brothers are also considered nearest neighbors. We denote by $\Lambda$ the set of all nodes of the tree and by $\mathcal{L}(\Lambda)$ the restriction of $\Lambda$ to the leaves. We apply this MR representation to the spatial part of the function $\mathbf{u}=(u, v)$, which corresponds to the numerical solution of the underlying problem for each time step, so we need to update the tree structure for the proper representation of the solution during the evolution. To this end, we apply the above thresholding strategy, but always ensure the graded tree structure of the data. Once the thresholding is performed, we add to the tree a safety zone, generated by adding one finer level to the tree in all leaves without violating the graded tree data structure. In addition, to enforce conservativity of the scheme, we compute only the fluxes at level $l+1$ and we set the ingoing flux on the leaf at 
level $l$ equal to the sum of the outgoing fluxes on the leaves of level $l+1$ sharing the same edge, i.e.

$$
F_{L^{l} \rightarrow K^{l}}=\sum_{S \in \mathcal{M}_{K^{l}},|\partial S \cap \partial L| \neq 0} F_{S^{l+1} \rightarrow T^{l+1},}
$$

where $T \notin \mathcal{M}_{K^{l}}$ is a control volume such that $|\partial T \cap \partial L| \neq 0$ and $T$ shares an edge with $S$.

\subsubsection{Data Compression Rate and Speed-up}

The quantity $\eta:=N /\left(2^{-(2 H)} N+\# \mathcal{L}(\Lambda)\right)$, denoted data compression rate [8], is used to measure the improvement in data compression. Here, $N$ is the number of control volumes in the full finest grid at level $l=H$, and $\# \mathcal{L}(\Lambda)$ is the number of leaves. The speed-up $\mathcal{V}$ between the CPU times of the numerical solutions obtained by the FV and MR methods is defined by $\mathcal{V}:=\mathrm{CPU}$ time $_{\mathrm{FV}} / \mathrm{CPU}$ time $_{\mathrm{MR}}$.

\subsection{Local Time Stepping}

To achieve further savings in computational time, we utilize the locally varying time stepping (LTS) device introduced by Müller and Stiriba [45]. It basically consists in enforcing a local stability condition by using the same CFL number for all levels, and then evolving all leaves on level $l$ using the local time step

$$
\Delta t_{l}=2^{H-l} \Delta t, \quad l=H-1, \ldots, 0,
$$

where $\Delta t=\Delta t_{H}$ corresponds to the time step on the finest level $l=H$, and for schemes constructed on the basis of Scheme A, this local stability condition will depend on the CFL condition given in (13). It is assumed that the fine grid is only used locally, so this strategy allows us to increase the time step for the major part of the adaptive mesh. In order to always have at hand the computed fluxes, we need to perform the locally varying time stepping recursively from fine to coarse levels. If at any instance of the procedure there is a missing value, we can project the value from the sons nodes or we can predict this value from the parent nodes. A more detailed description of the locally varying time stepping strategy is given in the following algorithm (see e.g. [5, 45]), a related technique using two step timeschemes was proposed in [16]. We here detail only the description for interior cells, and concentrate on the basis of Scheme B only. (The analogue algorithm based on Scheme A has already been applied to reaction-diffusion systems, see [5].) This algorithm is similar to Algorithm 2 in [45] and Algorithm 6.1 in [5]. The basic novelty is part (b) of step 2.

Algorithm 2 (Locally varying intermediate time stepping)

1. Grid adaptation (provided the former sets of leaves and virtual leaves).

2. do $k=1, \ldots, 2^{H}$ (intermediate time steps are $n+2^{-H}, n+2 \cdot 2^{-H}, n+3 \cdot 2^{-H}, \ldots$, $n+1)$
(a) Synchronization:
do $l=H, \ldots, 1$
if $1 \leqslant l \leqslant \tilde{l}_{k-1}$ then
if $K^{l}$ is a virtual leaf then compute kinetics by

$$
f_{K^{l}}^{n+k 2^{-H}} \leftarrow f_{K^{l}}^{n+(k-1) 2^{-H}}, \quad g_{K^{l}}^{n+k 2^{-H}} \leftarrow g_{K^{l}}^{n+(k-1) 2^{-H}}
$$




\section{endif}

else

if $K^{l}$ is a leaf then compute kinetics by

$$
\begin{aligned}
& f_{K^{l}}^{n+k 2^{-H}} \leftarrow \rho^{-1} u_{K^{l}}^{n+k 2^{-H}}\left(1-u_{K^{l}}^{n+k 2^{-H}}\right)\left(u_{K^{l}}^{n+k 2^{-H}}-a^{-1}\left[v_{K^{l}}^{n+k 2^{-H}}+b\right]\right) \\
& g_{K^{l}}^{n+k 2^{-H}} \leftarrow u_{K^{l}}^{n+k 2^{-H}}-v_{K, l}^{n+k 2^{-H}}
\end{aligned}
$$

endif

if $S^{l+1}, T^{l+1}$ are leaves (interface edges) then compute fluxes by

\section{endif}

$$
F_{L^{l} \rightarrow K^{l}} \leftarrow \sum_{S \in \mathcal{M}_{K^{l}},|\partial S \cap \partial L| \neq 0} F_{S^{l+1} \rightarrow T^{l+1}}
$$

\section{endif}

\section{enddo}

(b) Time evolution:

do $l=1, \ldots, H$

if $1 \leq l \leq \tilde{l}_{k-1}$ then there is no evolution:

$$
u_{K^{l}}^{n+(k+1) 2^{-H}} \leftarrow u_{K^{l}}^{n+k 2^{-H}}, \quad v_{K^{l}}^{n+(k+1) 2^{-H}} \leftarrow v_{K^{l}}^{n+k 2^{-H}}
$$

else

Interior marching formula only for the leaves $K^{l}$ :

Set $\operatorname{Lap}_{K^{l}}^{m}=0$ for all $m \in\{0,1\}\left(m^{\prime}=1-m\right)$.

if $u_{K^{l}}^{n+k 2^{-H}}<\delta$ then

$$
\begin{aligned}
& u_{K^{l}}^{n+(k+1) 2^{-H}} \leftarrow|K|^{-1} \Delta t_{l} \operatorname{Lap}_{K^{l}}^{m}, \quad v_{K^{l}}^{n+(k+1) 2^{-H}} \leftarrow\left(1-\Delta t_{l}\right) v_{K^{l}}^{n+k 2^{-H}} \\
& \text { else } \\
& \hat{u} \leftarrow \frac{1}{a} v_{K^{l}}^{n+k 2^{-H}}+\frac{b}{a}, \quad v_{K^{l}}^{n+(k+1) 2^{-H}} \leftarrow v_{K^{l}}^{n+k 2^{-H}}+\Delta t_{l}\left(u_{K^{l}}^{n+k 2^{-H}}-v_{K^{l}}^{n+k 2^{-H}}\right)
\end{aligned}
$$

if $\Delta t_{l} / \rho \leq 1$ then

else

$$
\begin{aligned}
u_{K^{l}}^{n+(k+1) 2^{-H}} \leftarrow & u_{K^{l}}^{n+k 2^{-H}}+|K|^{-1} \Delta t_{l} \operatorname{Lap}_{K^{l}}^{m} \\
& +\rho^{-1} \Delta t_{l} u_{K^{l}}^{n+k 2^{-L}}\left(1-u_{K^{l}}^{n+k 2^{-H}}\right)\left(u_{K^{l}}^{n+k 2^{-H}}-\hat{u}\right)
\end{aligned}
$$

if $u_{K^{l}}^{n+k 2^{-H}}<\hat{u}$ then

$$
\begin{aligned}
& u_{K^{l}}^{n+(k+1) 2^{-H}} \leftarrow|K|^{-1} \Delta t_{l} \operatorname{Lap}_{K^{l}}^{m} \\
& +u_{K^{l}}^{n+k 2^{-H}}\left[1-\rho^{-1} \Delta t_{l}\left(1-u_{K^{l}}^{n+k 2^{-H}}\right)\left(u_{K^{l}}^{n+k 2^{-H}}-\hat{u}\right)\right]^{-1}
\end{aligned}
$$

else

$$
\begin{aligned}
& \quad w \leftarrow \rho^{-1} \Delta t_{l} u_{K^{l}}^{n+k 2^{-H}}\left(u_{K^{l}}^{n+k 2^{-H}}-\hat{u}\right) \\
& \quad u_{K^{l}}^{n+(k+1) 2^{-H}} \leftarrow\left(u_{K^{l}}^{n+k 2^{-H}}+w\right)(1+w)^{-1}+|K|^{-1} \Delta t_{l} \operatorname{Lap}_{K^{l}}^{m} \\
& \text { endif }
\end{aligned}
$$

\section{endif}

$\operatorname{Lap}_{K^{l}}^{m^{\prime}} \leftarrow \operatorname{Lap}_{K^{l}}^{m^{\prime}}-\left|N\left(K^{l}\right)\right| A\left(u_{K^{l}}^{n+(k+1) 2^{-H}}\right)$

do for all $L \in N\left(K^{l}\right)$ (in this case, $L$ is at one of the levels $l-1, l$, or $l+1$ )

\section{enddo}

$$
\operatorname{Lap}_{L}^{m^{\prime}} \leftarrow \operatorname{Lap}_{L}^{m^{\prime}}+A\left(u_{K^{l}}^{n+(k+1) 2^{-H}}\right)
$$

\section{endif}

\section{endif}

$\operatorname{Lap}_{K^{l}}^{m} \leftarrow 0$

$m \leftrightarrow m^{\prime}$

\section{enddo}


(c) Partial grid adaptation each odd intermediate time step:

do $l=H, \ldots, \tilde{l}_{k}+1$

Projection from the leaves.

enddo

do $l=\tilde{l}_{k}, \ldots, H$

Thresholding, prediction, and addition of the safety zone.

\section{enddo \\ enddo}

Here, $\tilde{l}_{k}$ denotes the coarsest level containing leaves at the intermediate step $k$ (as introduced in [45]), $h(l)$ is the mesh size on level $l$. The interior marching formulas are obtained from Algorithm 1 for the intermediate time steps $k=1, \ldots, 2^{H}$, for the leaf in the position $K$ at level $l$.

\section{Numerical Results}

Our subsequent simulations will include comparisons based on error analysis. Therefore, and in the absence of exact solutions for these examples, we compute errors in different norms using a numerical solution on an extremely fine mesh as a reference solution. To measure errors between a reference solution $z_{\text {ref }}$ and an approximate solution $z_{h}$, at time $t^{n}$, we will use normalized $L^{p}$-errors:

$$
e_{p}^{n}=\frac{\left\|z_{\mathrm{ref}}^{n}-z_{h}^{n}\right\|_{p}}{\left\|z_{\mathrm{ref}}^{n}\right\|_{p}}, \quad p=1,2, \infty,
$$

where

$$
\begin{aligned}
\left\|z_{\mathrm{ref}}^{n}-z_{h}^{n}\right\|_{\infty} & =\max _{K \in \mathcal{T}}\left|z_{\mathrm{ref}, K}^{n}-z_{h_{K}}^{n}\right| \\
\left\|z_{\mathrm{ref}}^{n}-z_{h}^{n}\right\|_{p} & =\left(\frac{1}{|K|} \sum_{K \in \mathcal{T}}\left|z_{\mathrm{ref}, K}^{n}-z_{h_{K}}^{n}\right|^{p}\right)^{1 / p}, \quad p=1,2 .
\end{aligned}
$$

Here $z_{\text {ref, } K}^{n}$ stands for the projection of the reference solution onto the control volume $K$. Convergence rates for a scalar field $z$ are calculated by $r(z)=\log \left(e(z) / e^{*}(z)\right)\left[\log \left(h / h^{*}\right)\right]^{-1}$, where $e(z)$ and $e^{*}(z)$ denote the respective errors computed for two consecutive meshes of sizes $h$ and $h^{*}$. Eventually, we will also need to compute errors between a reference solution and an approximate solution obtained using multiresolution. In this case, $z_{h K}^{n}$ in (22) stands for the approximate MR solution on the cell $K$ at the finest resolution level $l=H$. This value is obtained by applying a prediction procedure from the corresponding leaf whenever the leaf is not at level $l=H$.

\subsection{Examples 1 and 2}

For our first pair of examples, which are included to illustrate the effect of degenerate diffusion independently of the MR device, we consider a simple square domain $\bar{\Omega}=[0,180]^{2}$ with the following model parameters chosen according to [2]: $N=200^{2}, a=0.75, b=$ 

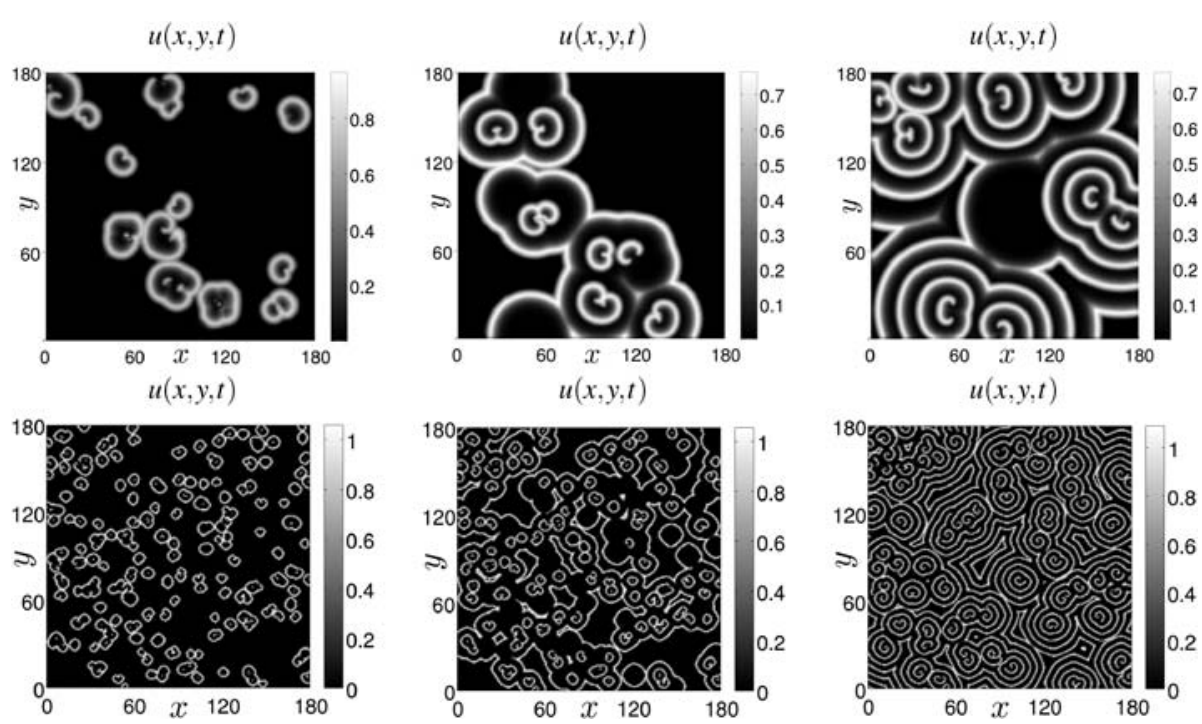

Fig. 2 Examples 1 and 2: Recovery fronts for the Barkley model (1), (5) with a constant diffusion coefficient (2) (top) and degenerate diffusion (3) (bottom) at times $t=9$ (left), $t=18$ (middle) and $t=27$ (right), simulated by Scheme B on a uniform grid

Table 1 Example 1: number of control volumes, meshsize $h$, normalized approximate $L^{1}$-errors $e_{1}^{n}$ for the recovery variable $u$ and observed convergence rates $r^{1}(u)$ at simulated time $t=10$ for Schemes A and B

\begin{tabular}{cllllll}
\hline Mesh & \multicolumn{3}{l}{ Scheme A } & & \multicolumn{2}{l}{ Scheme B } \\
& $h$ & $e_{1}^{n}$ & $r^{1}(u)$ & & $r_{1}^{n}(u)$ \\
\hline $80 \times 80$ & 1.8750 & $2.49 \times 10^{-2}$ & - & & $6.86 \times 10^{-2}$ & - \\
$200 \times 200$ & 0.7500 & $3.60 \times 10^{-3}$ & 1.2416 & $9.37 \times 10^{-3}$ & 1.0974 \\
$400 \times 400$ & 0.3750 & $5.61 \times 10^{-4}$ & 1.1647 & $9.91 \times 10^{-4}$ & 1.1866 \\
$800 \times 800$ & 0.1875 & $9.05 \times 10^{-5}$ & 1.1309 & & $1.42 \times 10^{-4}$ & 1.2007 \\
\hline
\end{tabular}

0.02, $\rho=0.02, \Delta t=180^{2} /\left\{5(N-1)^{2}\right\}$, and $D=1$. In Example 1, we utilize the standard diffusion function (2), and in Example 2, the degenerate diffusion function (3) with $u^{*}=0.75$. The initial values for both $u$ and $v$ are uniformly distributed for the entire domain, however, as discussed in e.g. [65], for patterns to appear, it is sufficient to consider constant initial data perturbed only on a small portion of the domain. The computation is done without multiresolution, and Fig. 2 shows snapshots of the numerical solution for $u$ obtained by using Scheme B with $\delta=1 \times 10^{-3}$ on a uniform mesh. It can be seen that the recovery fronts are formed by two-armed spiral waves. Moreover, we observe that the solution for Example 2 exhibits a larger number of spirals, which in turn have sharper fronts.

We select Example 1 for comparison with approximate solutions obtained by Scheme A. Table 1 illustrates that the approximate errors obtained for Scheme B (i.e., the distance to a reference solution) are slightly larger than those obtained with Scheme A (the reference solution is also computed by Scheme A, which may in part explain this "bias"), but still comparable. Moreover, the approximate convergence rates obtained by both methods are also comparable, both reaching $\mathcal{O}(h)$. Specifically, an order of approximately $\alpha=1.2$ is 

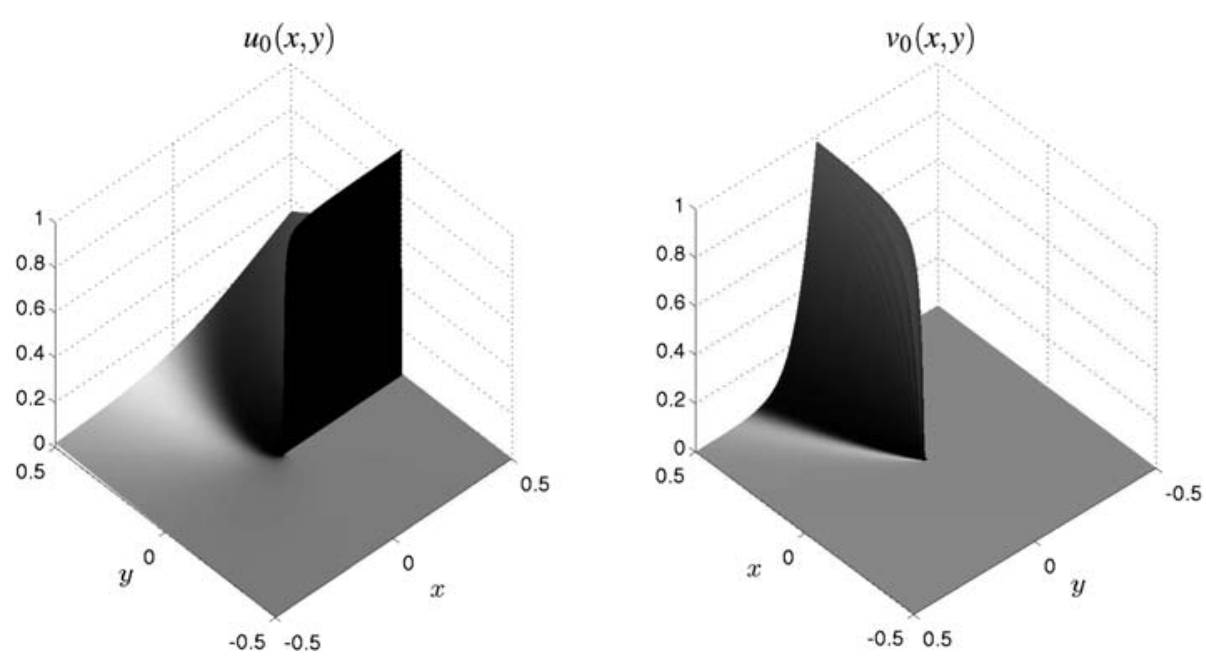

Fig. 3 Example 3: initial data for the Barkley model according to [62]

attained, and this value is also needed in the computation of the threshold parameter $\varepsilon_{\mathrm{R}}$ for MR computations, to be used in the following examples. We also mention that there exists a notable difference in speed-up between both Schemes A and B. For instance, the CPU time required to advance the approximate solution until $t=10$ has been determinated, and Scheme B is about four times faster than Scheme A.

\subsection{Example 3}

In order to understand the differences between the dynamics of both components of the solution, we now perform in Example 3 a computation using the MR scheme, called Scheme A-MR, which is based on Scheme A as the underlying reference scheme. We select parameters according to Weiser [62], namely $\bar{\Omega}=[-0.5,0.5]^{2}, D=0.002, \rho=0.01, b=0.01$, and $a=0.8$. To initiate the formation of spiral waves, we choose the initial functions

$$
u_{0}(r, \theta):=\tanh (5 \theta) \phi(r), \quad v_{0}(r, \theta):=\tanh (5 \theta) \phi(r+0.5)
$$

(see Fig. 3), where

$$
\begin{aligned}
& 0<r=\sqrt{x^{2}+y^{2}}<0.5, \quad 0<\theta=\arctan (y / x)<2 \pi, \\
& \phi(\theta):=\sum_{k \in \mathbb{Z}} \exp \left(-2(2 \pi k+\theta)^{2}\right) .
\end{aligned}
$$

We use $H=8$ resolution levels for the MR analysis, and as a result of a series of test computations we choose the nearly optimal reference tolerance $\varepsilon_{\mathrm{R}}=3.5 \times 10^{-4}$ (more details on the procedure to obtain the value for $\varepsilon_{\mathrm{R}}$ are provided for Example 4). Figure 4 shows snapshots of both components of the numerical solution along with the adaptive mesh. Figure 5(a) shows a profile of the approximate solution at $y=0.25$ (also for both components $u$ and $v$ ), for the particular time $t=20$, along with its corresponding tree structure plotted in Fig. 5(b). Even though it corresponds to a slice of the spiral wave solution of Fig. 4, this profile is similar to the behaviour of one-dimensional planar waves. The transition from the 

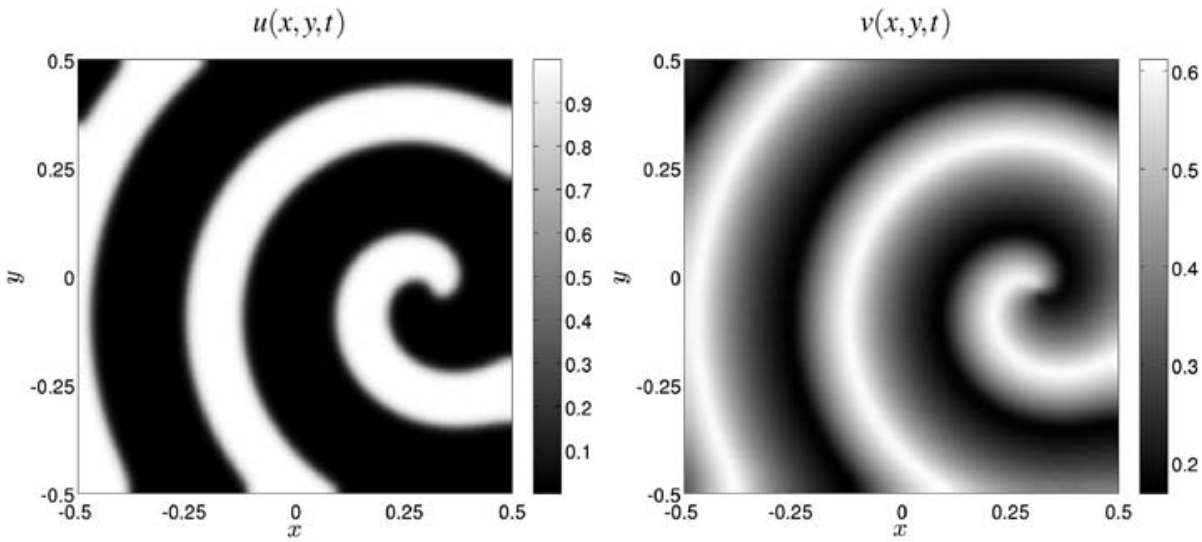

Positions of the leaves

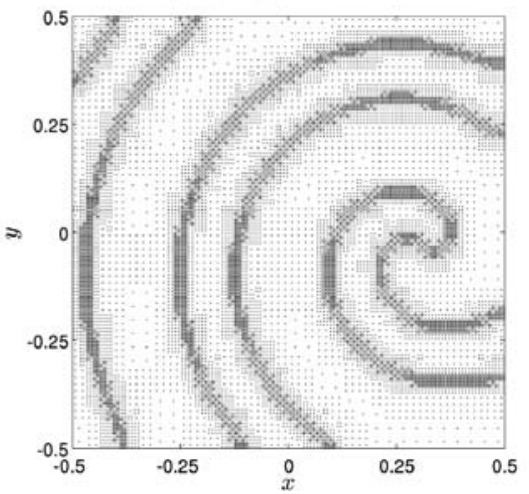

Fig. 4 Example 3: numerical solution of $u$ and $v$ together with the corresponding adaptive mesh for the Barkley model at time $t=20$, calculated by Scheme A-MR with $H=8$ MR levels

(a)

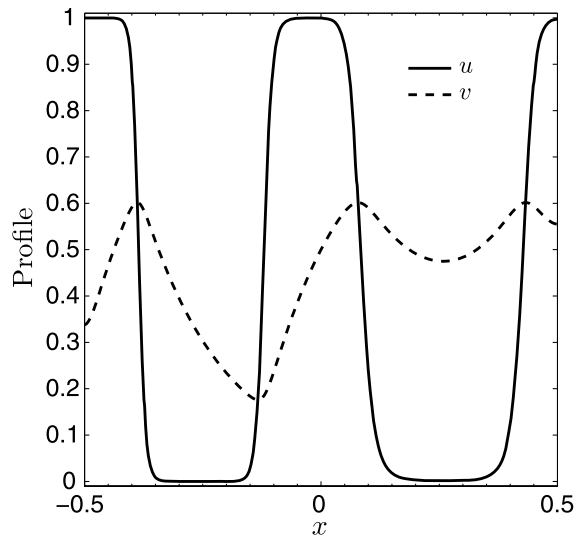

(b)

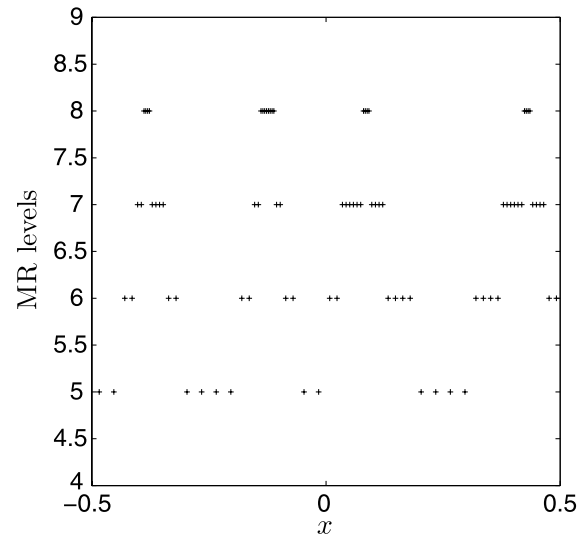

Fig. 5 Example 3: (a) profile of the numerical solution at $y=0.25$ and (b) corresponding adaptive mesh for the Barkley model at time $t=20$, calculated by Scheme A-MR 
(a)

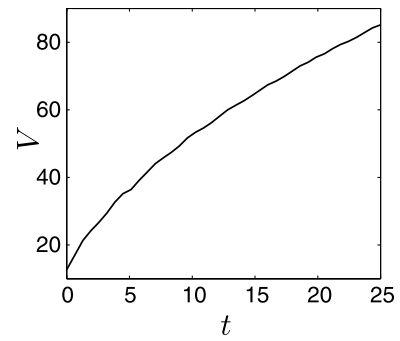

(b)

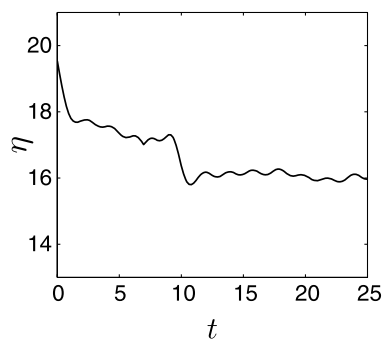

(c)

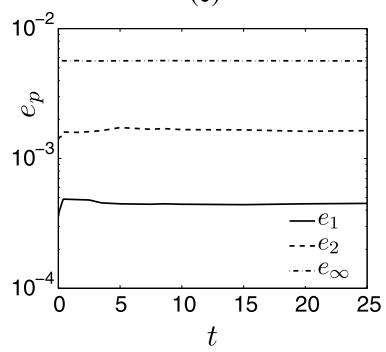

Fig. 6 Example 3: time evolution of the (a) speed-up rate, (b) data compression rates, and (c) normalized errors for the scheme A-MR

(a)

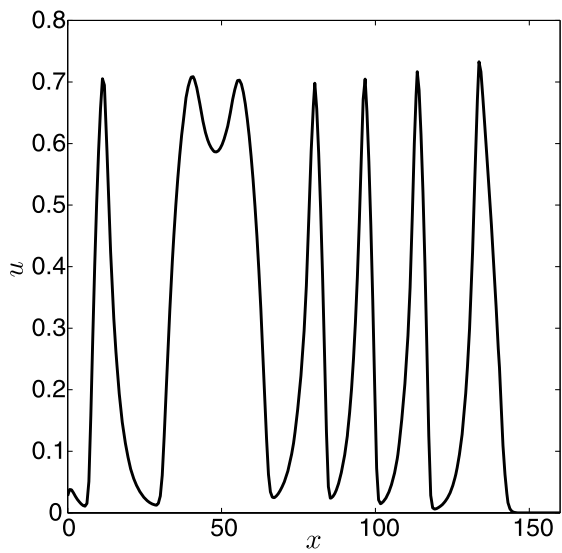

(b)

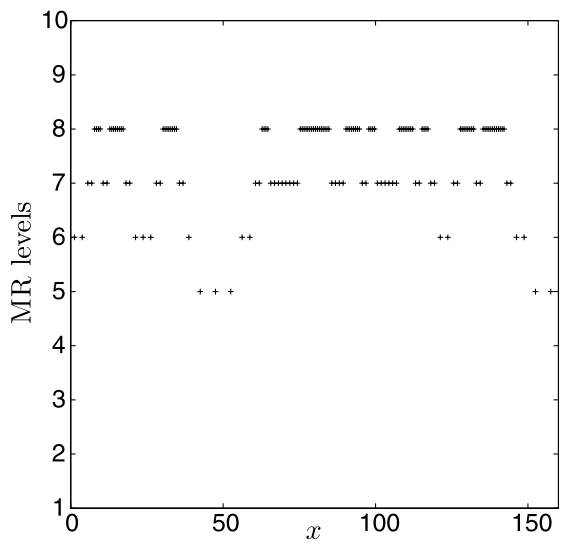

Fig. 7 Example 4: (a) profile of the recovery front and (b) corresponding tree at level $y=120$ and time $t=50$, calculated by Scheme B-MR-LTS with $\delta=1.0 \times 10^{-3}, H=8$ resolution levels and $\varepsilon_{\mathrm{R}}=1.12 \times 10^{-3}$

resting state $u \approx 0$ to the excited state $u \approx 1$ is a sharp interface propagating through the excitable medium, as should be expected since the diffusion parameter $D$ is fairly small. In contrast to this, the "slow" field $v$ shows a much smoother transition. Furthermore, the MR scheme captures accurately both fast and slow scale features, using the maximum resolution only on small portions of the domain. Here, we also present the evolution in time of the compression rates $\mathcal{V}$ and $\eta$ and normalized errors (see Fig. 6).

\subsection{Example 4}

In Fig. 9 we display the numerical solution computed by Scheme B applying MR and locally varying time stepping (scheme B-MR-LTS). The solution is plotted along with the corresponding positions of the leaves that form the adaptive mesh. For this case (Example 4), the parameters are the same as in Example 1, except for $\bar{\Omega}=[0,160]^{2}$ and $N=256^{2}$. The width of the recovery fronts can be seen from a transversal profile of the solution at level $y=120$ with its corresponding tree structure, which is shown in Fig. 7.

For the MR setting we use $H=8$ resolution levels. Now, for obtaining an optimal reference tolerance $\varepsilon_{\mathrm{R}}$, a more careful consideration needs to be done. Clearly, as is mentioned 
in Sect. 3.1.2, choosing a very small threshold parameter would imply an unnecessary refinement, and an overestimation of the tolerance would imply larger errors. To obtain $\varepsilon_{\mathrm{R}}$ from (21), we need to determine the factor $C$ based on preliminary computations involving error analysis and comparison of data compression rate $\eta$ and CPU speed-up $\mathcal{V}$. Basically, we select the largest value of $C$ (among a finite number of test values) such that both compression rates are maximized while the errors are controlled to have the same slopes as the reference scheme (A or B). This preliminary study needs to be done just once, and in order to minimize the required computational effort, we perform these calculations for an auxiliary one-dimensional problem, which is obtained by fixing a level ( $y=120$ in our case). Despite the possible differences between the 2D solution and the planar travelling wave, it is possible to capture with sufficient accuracy the essential features of the local solution structure (see e.g. [62]). In practice, we extract a slice of the numerical solution of the two-dimensional problem at time $t=49.5$. Then we use this as initial condition for the one-dimensional version of (1) and we evolve such a system until we reach $t=50$.

A snapshot of the numerical one-dimensional solution at time $t=50$ is presented in Fig. 7, and the preliminary computations to determine the factor $C$ are summarized in Fig. 8. Note that the value $C=2.5 \times 10^{4}$ yields the optimal threshold $\varepsilon_{\mathrm{R}}=1.12 \times 10^{-3}$, which corresponds to $\varepsilon_{\mathrm{R}} \approx \delta$ for the two-dimensional problem. Since $\delta$ is the size of the boundary layer related to the $u$-nullcline of the system, following the construction of Scheme $\mathrm{B}$, it is clear that details that are below $\delta$ will be not considered neither by the MR device nor by the reference scheme $\mathrm{B}$. Then, an additional way of obtaining savings in computational effort, would be to simply set $\varepsilon_{\mathrm{R}}=\delta$, avoiding the computation of $\varepsilon_{\mathrm{R}}$ using (21).

A comparison of the corresponding schemes has been carried out, and from Table 2 it is clear that the adaptive method based on Scheme A (Scheme A-MR-LTS) yields slightly

(a)

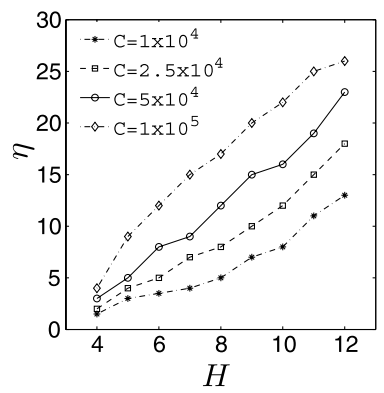

(b)

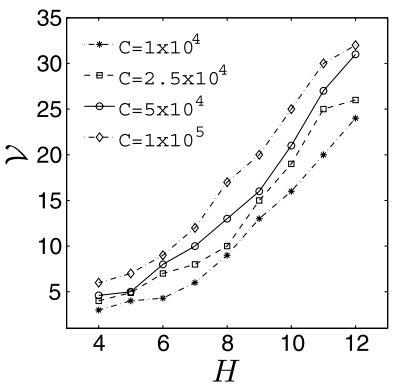

(c)

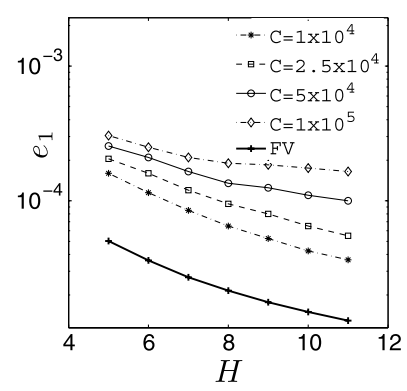

Fig. 8 Example 4: determination of the factor $C$ in (21). (a) Data compression rate $\eta$, (b) Speed-up factor $\mathcal{V}$, and (c) normalized $L^{1}$-errors, for different levels $H$ and values of $C$ at time $t=50$

Table 2 Example 4: Corresponding simulated time, used numerical scheme, speed-up rate $\mathcal{V}$, compression rate $\eta$ and normalized errors for the recovery variable computed with respect to a reference solution obtained by Scheme A on a fine mesh of $2^{2 \cdot 11}=2048^{2}=4194304$ control volumes

\begin{tabular}{lllllll}
\hline Time & Scheme & $\mathcal{V}$ & $\eta$ & $e_{1}^{n}$ & $e_{2}^{n}$ & $e_{\infty}^{n}$ \\
\hline \multirow{2}{*}{$t=20$} & A-MR-LTS & 15.36 & 21.64 & $1.24 \times 10^{-4}$ & $2.42 \times 10^{-5}$ & $3.63 \times 10^{-4}$ \\
& B-MR-LTS & 48.21 & 22.31 & $4.63 \times 10^{-4}$ & $3.51 \times 10^{-5}$ & $4.97 \times 10^{-4}$ \\
$t=50$ & A-MR-LTS & 19.49 & 11.42 & $3.60 \times 10^{-4}$ & $5.63 \times 10^{-5}$ & $1.07 \times 10^{-3}$ \\
& B-MR-LTS & 57.25 & 11.39 & $5.13 \times 10^{-4}$ & $7.29 \times 10^{-5}$ & $9.64 \times 10^{-4}$ \\
\hline
\end{tabular}




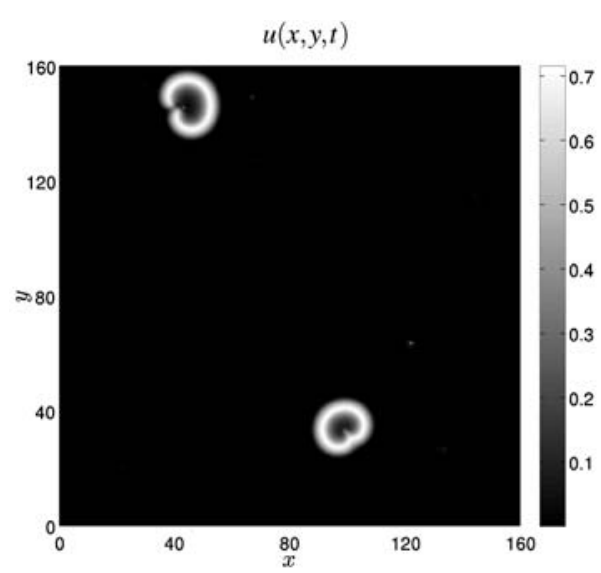

Positions of the leaves

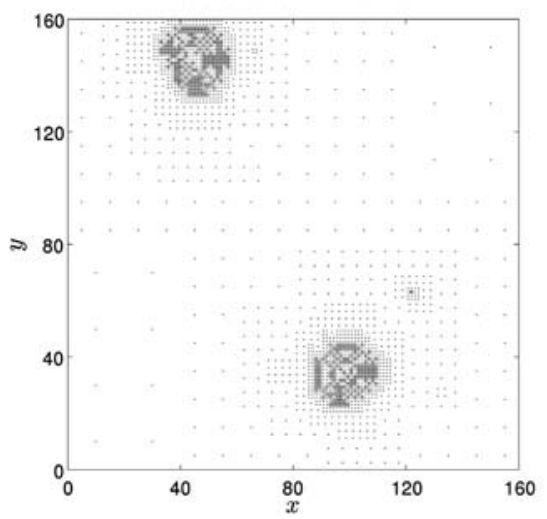

$u(x, y, t)$

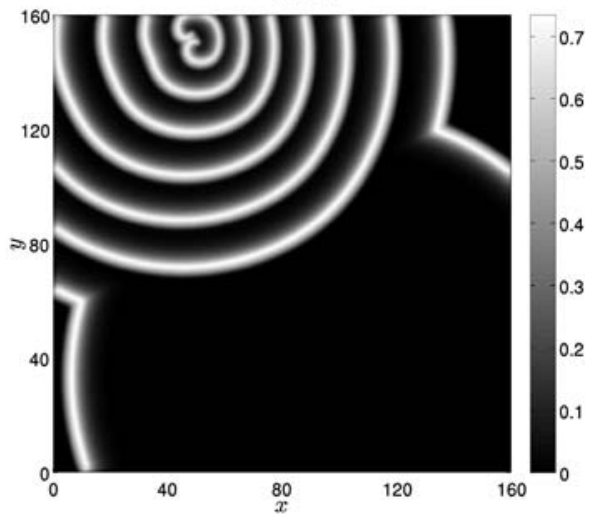

Positions of the leaves

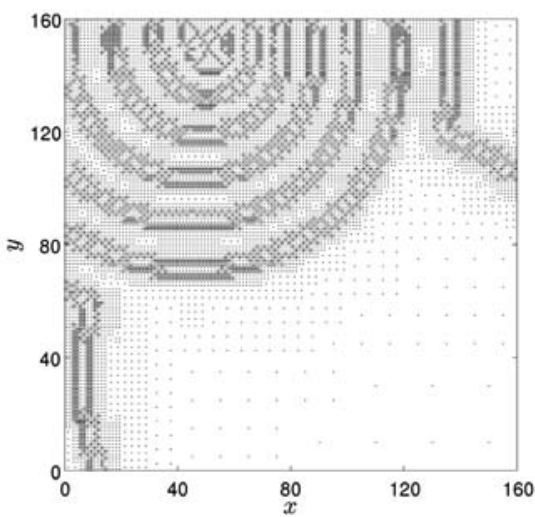

Fig. 9 Example 4: Recovery fronts and leaves of the tree data structure at times $t=20$ (left), $t=50$ (right). $H=8$ resolution levels and $\varepsilon_{\mathrm{R}}=\delta=1 \times 10^{-3}$, calculated by Scheme B-MR-LTS

smaller error levels than those based on Scheme B (Scheme B-MR-LTS). This is not surprising since we are comparing against a reference solution computed by Scheme A. We can also notice that the data compression rate is very similar for all times for both methods, implying that the evolving meshes used by the methods are essentially the same. However, when using Scheme B-MR-LTS, a substantial improvement is seen in the speed-up. Even though, such gain in speed-up over the A-MR-LTS scheme is only of about three times and not four, as reported in Example 1 when comparing Schemes A and B. This apparent inconsistency could be explained, however, by the sub-linear growth of $\mathcal{V}$ induced by the MR strategy (as reported in Fig. 6(a)).

A difficult task is to compare solutions of systems starting from randomly distributed initial data. In practice, we compute errors as follows. The system is evolved until the "random noise" which is imposed as an initial condition on the finest grid has been smoothed sufficiently and has been organized into wave-like structures; then, the solution is projected on coarser levels to obtain auxiliary initial conditions for all levels required. For example, for the Scheme B-MR-LTS, the desired smoothing is reached within 100 time steps. This initial smoothing of the noise is also needed when computing MR solutions, since thresholding 

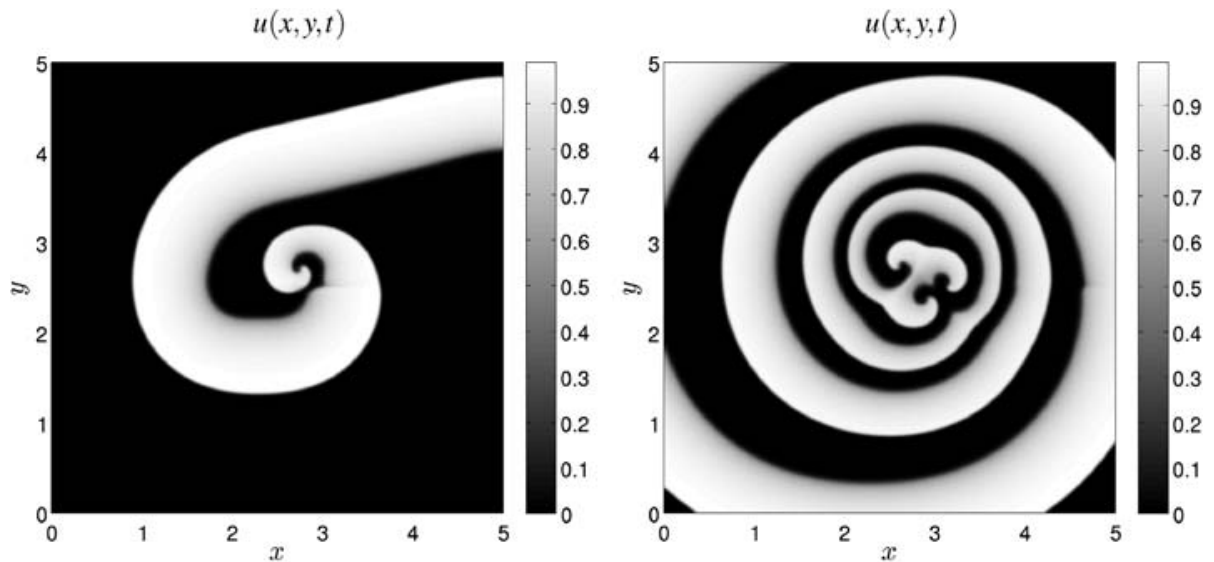

Positions of the leaves

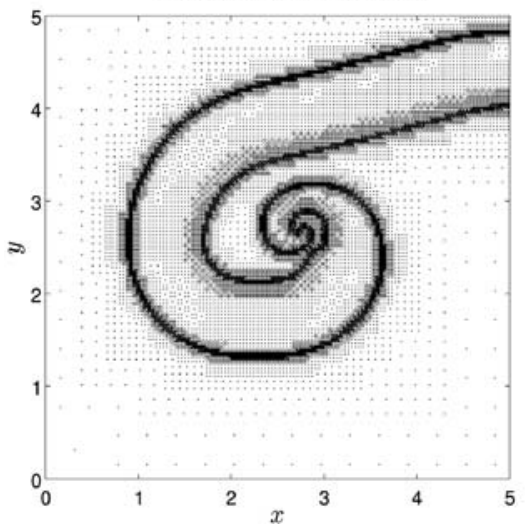

Positions of the leaves

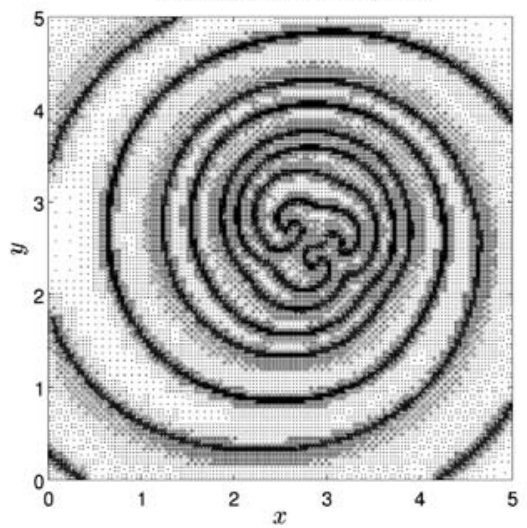

Fig. 10 Example 5: recovery fronts and adaptive tree structures at times $t=75$ (left) and $t=150$ (right) calculated by Scheme A-MR-LTS. Here $H=9$ resolution levels are used with a reference tolerance set to $\varepsilon_{\mathrm{R}}=5.5 \times 10^{-4}$

initial data with noise would discard significant information and therefore the computations on the regular fine mesh and the adaptive one will have spurious differences.

\subsection{Example 5}

In this example, we utilize the model (1), (2) with $D=0.01$ and employ the Aliev-Panfilov kinetics (6) with the parameters $a=b=0.1, k=8.0, c=0.01, d_{1}=0.12$, and $d_{2}=0.3$. The approximate solution is obtained by Scheme A-MR-LTS, for which the discretization parameters are $H=9$ resolution levels, reference tolerance $\varepsilon_{\mathrm{R}}=5.5 \times 10^{-4}, \bar{\Omega}=[0,5]^{2}$, $N=512^{2}$. A re-entrant wave is generated using a wavefront as initial data, which after $t=25$ is broken at the center of the domain (external stimulus $I_{\text {ext }}$ ). Next, the resulting electrical activity (field $u$ ) over the homogeneous excited domain and its corresponding adaptive mesh are illustrated in Figs. 10 and 11. After approximately 10 re-entrant rotations (which occurs at $t \approx 100$ ), spiral break-up becomes evident near the core, and it will lead the system to a state that displays spatiotemporal chaos and spiral turbulence. Approximate errors and compression rates are depicted in Table 3. It is apparent that as long as the solution 

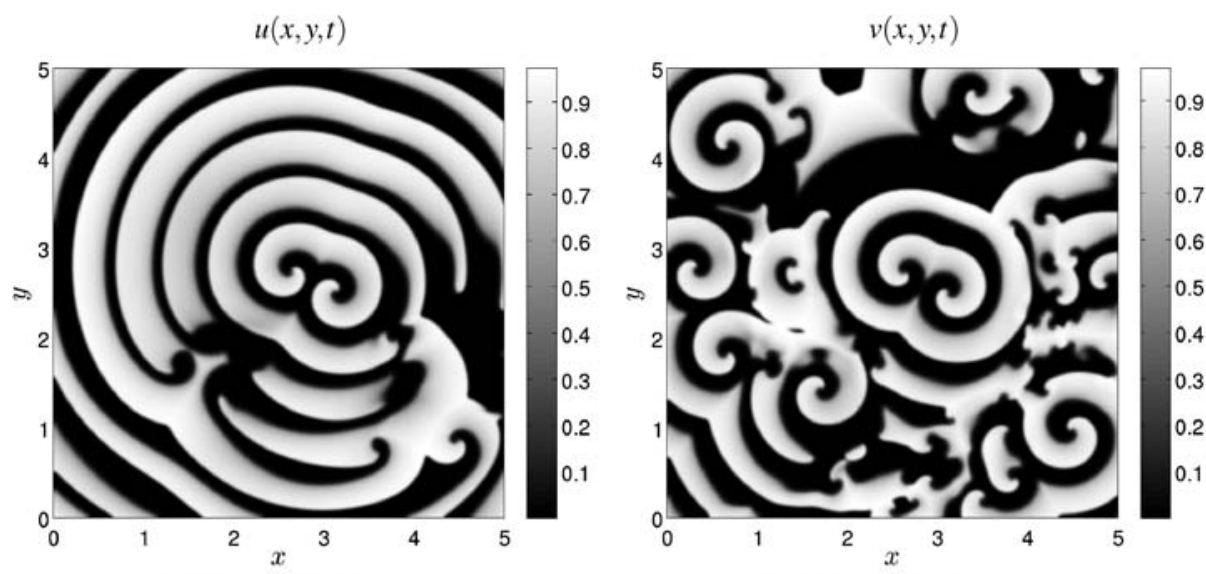

Positions of the leaves

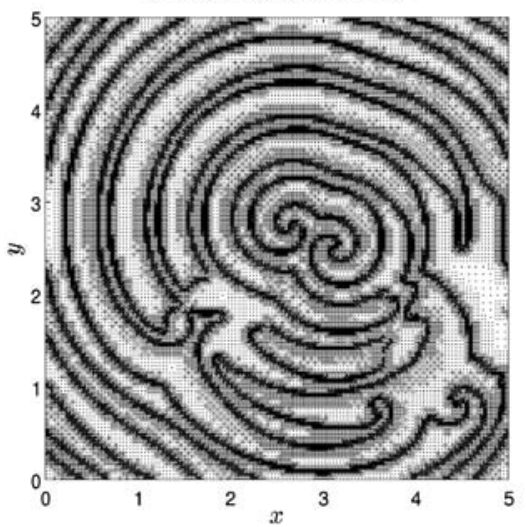

Positions of the leaves

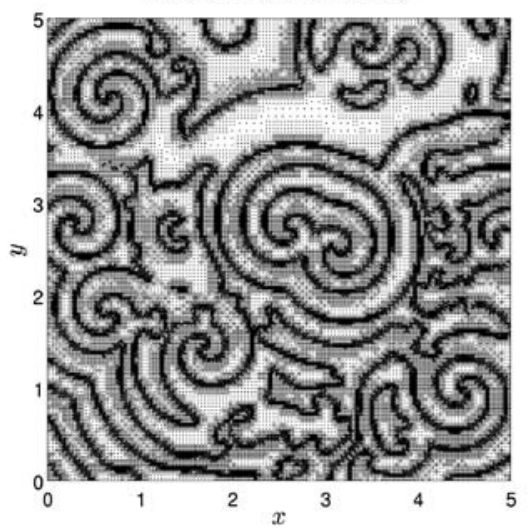

Fig. 11 Example 5: recovery fronts and adaptive tree structures at times $t=600$ (left) and $t=1000$ (right) calculated by Scheme A-MR-LTS. Here $H=9$ resolution levels are used with a reference tolerance set to $\varepsilon_{\mathrm{R}}=5.5 \times 10^{-4}$

Table 3 Example 5: Computation using Scheme A-MR-LTS. Corresponding simulated time, speed-up rate $\mathcal{V}$, compression rate $\eta$ and normalized errors for the recovery variable calculated with respect to a reference solution obtained by Scheme A on a fine mesh of 4194304 control volumes

\begin{tabular}{llllll}
\hline Time & $\mathcal{V}$ & $\eta$ & $e_{1}^{n}$ & $e_{2}^{n}$ & $e_{\infty}^{n}$ \\
\hline$t=75$ & 16.12 & 26.87 & $3.15 \times 10^{-4}$ & $8.47 \times 10^{-5}$ & $6.11 \times 10^{-4}$ \\
$t=100$ & 29.41 & 22.88 & $5.04 \times 10^{-4}$ & $9.62 \times 10^{-5}$ & $6.87 \times 10^{-4}$ \\
$t=150$ & 43.70 & 19.62 & $5.91 \times 10^{-4}$ & $1.33 \times 10^{-4}$ & $7.90 \times 10^{-4}$ \\
$t=300$ & 61.02 & 19.01 & $5.89 \times 10^{-4}$ & $1.64 \times 10^{-4}$ & $8.61 \times 10^{-4}$ \\
$t=500$ & 63.33 & 17.18 & $7.35 \times 10^{-4}$ & $2.17 \times 10^{-4}$ & $1.84 \times 10^{-3}$ \\
$t=1000$ & 68.15 & 15.77 & $1.04 \times 10^{-3}$ & $2.23 \times 10^{-4}$ & $1.97 \times 10^{-3}$ \\
\hline
\end{tabular}

structure becomes irregular, the data compression rate decreases and the MR looses its effectiveness. Nevertheless, it is also readily observed the MR-LTS strategy allows savings around 60 times in terms of speed-up while the errors remain controlled. 


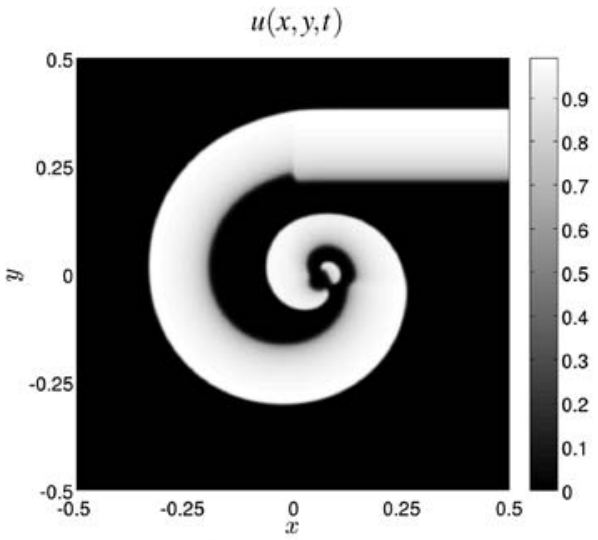

Positions of the leaves

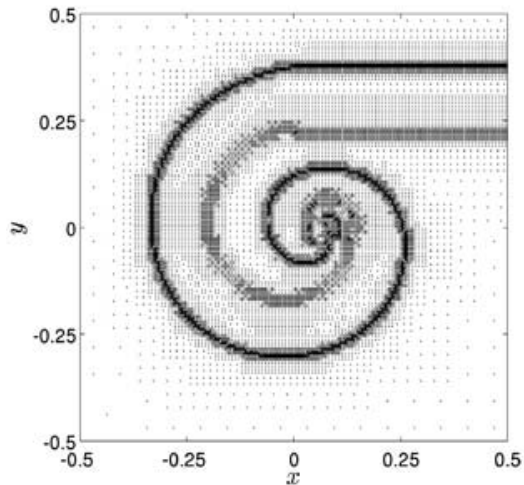

$u(x, y, t)$

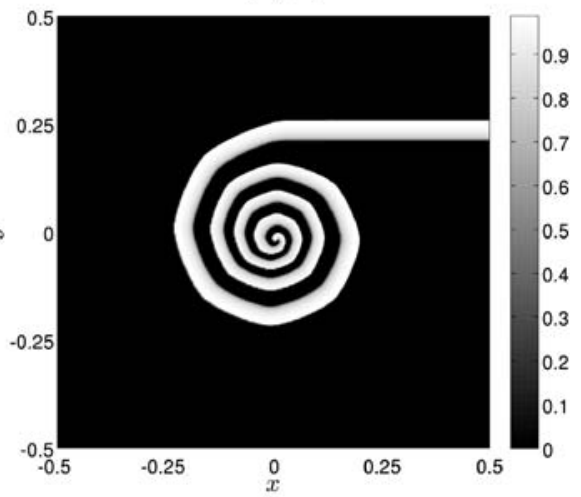

Positions of the leaves

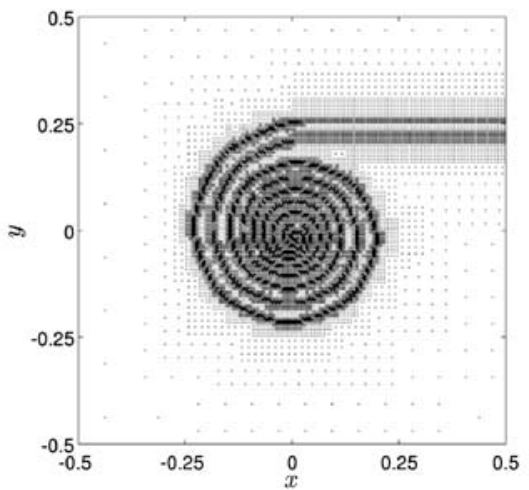

Fig. 12 Examples 6 and 7: Recovery fronts and adaptive tree structures at time $t=80$ calculated by Scheme A-MR-LTS using constant (left) and strongly degenerate diffusion (right). Here $H=9$ resolution levels are used with the reference tolerance $\varepsilon_{\mathrm{R}}=5.5 \times 10^{-4}$

\subsection{Examples 6, 7 and 8}

For our last examples we consider (1) with a standard diffusion coefficient (2) and alternatively a degenerating diffusion coefficient (3), where $f$ and $g$ are chosen according to the Aliev-Panfilov kinetics (6) with the parameters $a=b=0.1, k=8.0, c=0.01, d_{1}=0.12$, $d_{2}=0.3, D=0.01, \bar{\Omega}=[-0.5,0.5]^{2}$, as in Example 5. However, in contrast to that example, we here do not apply an external stimulus, i.e., we set $I_{\mathrm{ext}}=0$. Instead, the initial conditions are explicitly given by

$$
\begin{aligned}
& u_{0}(x, y)= \begin{cases}1 & \text { for } x \geq 0 \text { and }-0.01 \leq y<0, \\
0 & \text { otherwise },\end{cases} \\
& v_{0}(x, y)= \begin{cases}1 & \text { for } x<0, \\
1.5 & \text { for } x \geq 0 \text { and } y<-0.01, \\
-0.5 y & \text { for } x \geq 0 \text { and }-0.01 \leq y<0, \\
0 & \text { otherwise. }\end{cases}
\end{aligned}
$$




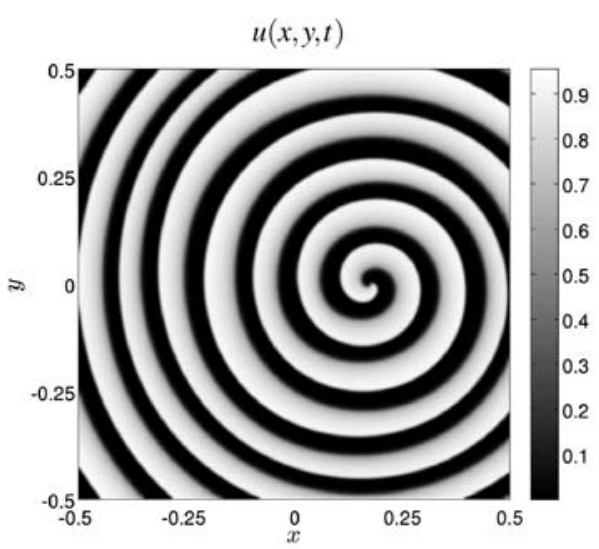

Positions of the leaves

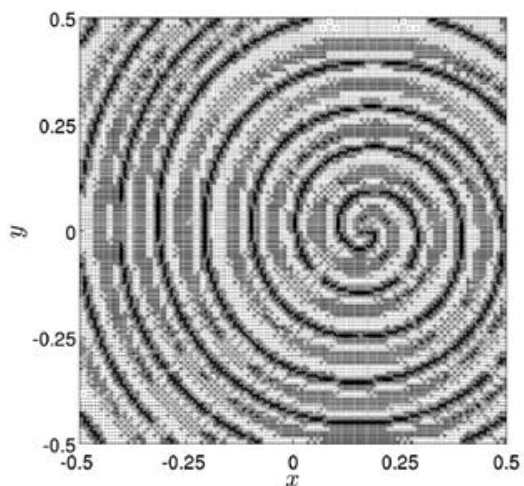

$u(x, y, t)$

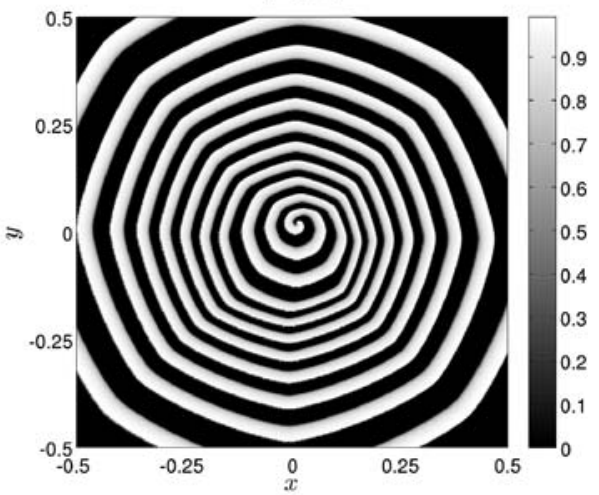

Positions of the leaves

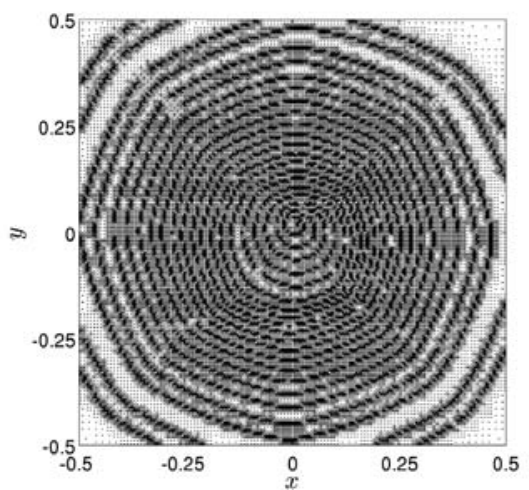

Fig. 13 Examples 6 and 7: Recovery fronts and adaptive tree structures at time $t=500$ calculated by Scheme A-MR-LTS using constant (left) and strongly degenerate diffusion (right). Here $H=9$ resolution levels are used with a reference tolerance set to $\varepsilon_{\mathrm{R}}=5.5 \times 10^{-4}$

To ensure that the qualitative features we find are not produced by other artifacts, we perform two simulations that only differ in the choice of $A(u)$. First, in Example 6, we solve the problem for a constant diffusion coefficient (2) (which means that the conductivity is the same through the entire medium), and next, in Example 7, we use the strongly degenerate diffusion term (3) with $u^{*}=0.85$. This may correspond to regarding the excitable medium as for example being injured by the effect of past arrhythmias [57], and not being able to propagate correctly the electrical potential when this is below the value $u^{*}=0.85$ (we do not choose a lower value since typically the repolarization of $u$ involves a jump of 80 $95 \%$ [20]). Roughly speaking, from the viewpoint of the equation, diffusion is "turned on" at the threshold $u^{*}$. This effectiveness of diffusion on a smaller $u$-interval is expected to exhibit sharper fronts than in the nondegenerate case. The approximate solution is obtained by scheme A-MR-LTS, for which the discretization parameters are $H=9$ resolution levels, the reference tolerance $\varepsilon_{\mathrm{R}}=5.5 \times 10^{-4}$, and $N=512^{2}$.

We stress that neither the well-posedness analysis and convergence of the numerical solutions obtained by Scheme A (and therefore for scheme A-MR-LTS) are covered for the model with degenerate diffusion (1), (3), nor is the well-posedness of (1), (3) itself covered in the case of zero-flux boundary conditions. In fact, significant advances have been made in 

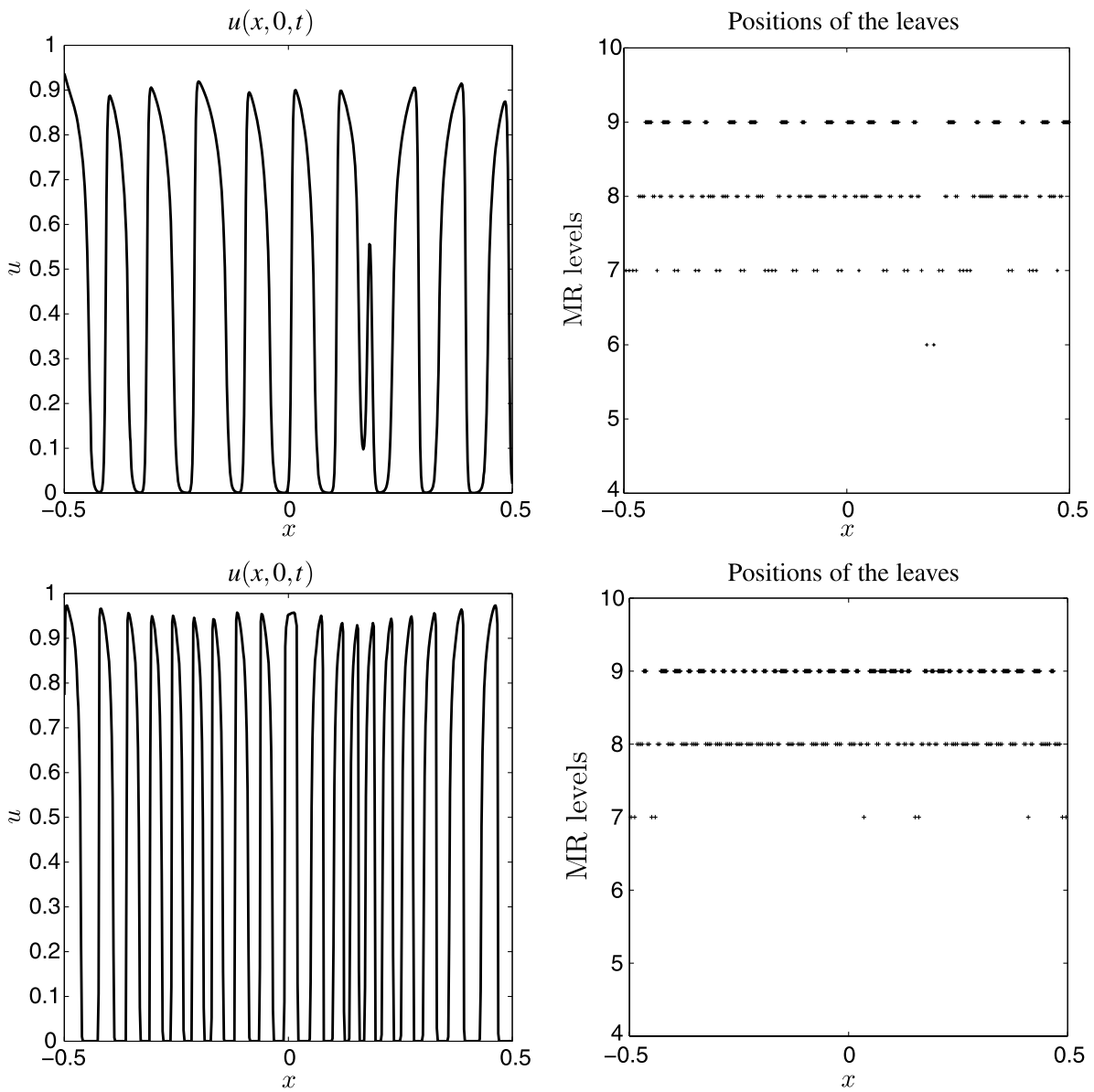

Fig. 14 Examples 6 and 7: profile view at $y=0$ of the recovery fronts and corresponding adaptive tree structures at time $t=500$ calculated by Scheme A-MR-LTS using constant (top) and strongly degenerate diffusion (bottom). Here $H=9$ resolution levels are used with a reference tolerance set to $\varepsilon_{\mathrm{R}}=5.5 \times 10^{-4}$

the well-posedness analysis (see e.g. [9, 40]) and the numerical analysis [19, 34, 41] of scalar strongly degenerate parabolic equations. The well-posedness of weakly coupled systems of such equations, of which (1), (3) is a special case, has, however, only been established for initial value problems (without boundary conditions) [30].

The numerical results are provided in Figs. 12 and 13. Although the approximate solutions obtained with and without degenerate diffusion are obviously different, a qualitative comparison is possible from Fig. 14, where it can be noticed that as expected, the fronts obtained with model (1), (3) are indeed sharper.

The spirals produced in the strongly degenerate parabolic case (Example 7), which are shown in the right plots of Figs. 12 and 13, have a close-to-octagonal shape which is aligned with the $(x, y)$-coordinate system. This observation raised the question whether the octagonal shape is possibly a numerical artefact associated with the Cartesian computational mesh. To check this, we performed another numerical experiment (Example 8) based on the same reaction-diffusion equations as in Example 7, but considering a new computational domain 

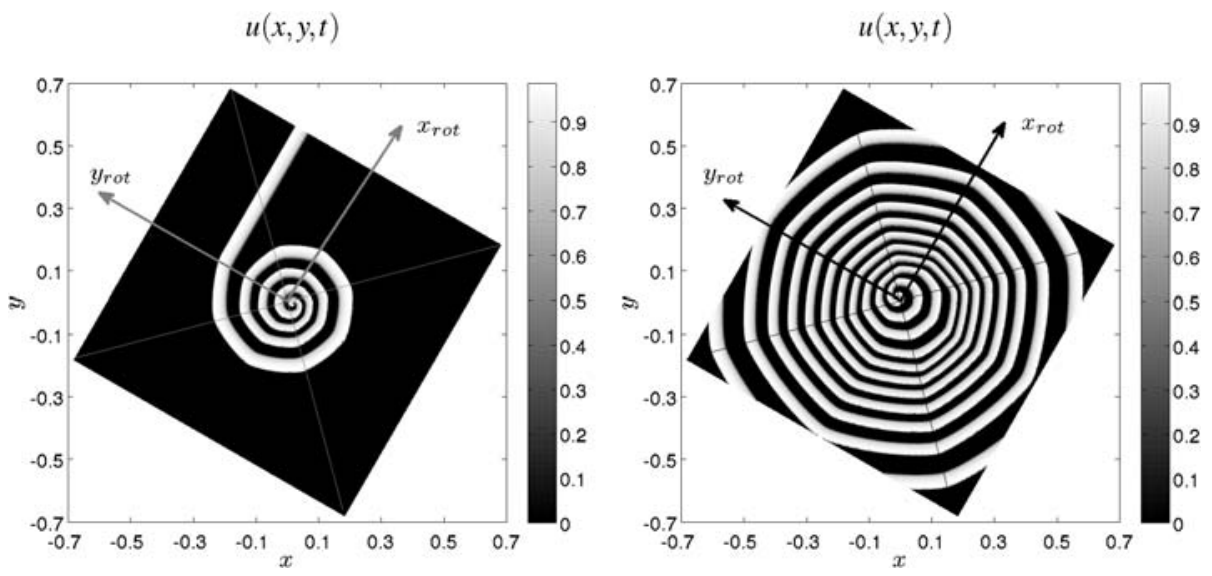

Fig. 15 Example 8: Recovery fronts at times $t=80$ and $t=500$ for the strongly degenerate diffusive case, calculated by Scheme A-MR-LTS on a domain rotated in counter-clockwise sense by an angle of $\pi / 3$. The Cartesian computational grid is aligned with the $x$ - and $y$-axes

$\bar{\Omega}$ obtained by rotating the domain $\Omega$ by an angle of $\pi / 3$ in the counter-clockwise sense. The initial datum (23) is rotated correspondingly. The (adaptive) computational grid, however, for Scheme A-MR-LTS is kept aligned with the $x$ - and $y$-axes. Figure 15 shows the numerical result. The directions $x_{\text {rot }}$ and $y_{\text {rot }}$ indicate the orientation of the rotated domain $\bar{\Omega}$. We again obtain spirals of close-to-orthogonal shape. However, as we emphasize by some thin straight lines in both plots of Fig. 15, the axes of "symmetry" of the spirals are aligned with the orientation of $\bar{\Omega}$. They are not aligned with the $x$ - and $y$-axes of the Cartesian computational grid. In other words, the numerical results of Example 8 are basically rotated versions of those of Example 7. Thus, we conclude that the close-to-octagonal shape of the spirals in Figs. 12 and 13 is not a numerical artefact, but is rather associated with the shape of domain $\Omega$ and the initial condition, and particular solution behaviour under degenerate diffusion.

\section{Conclusion}

A new fully space-time adaptive MR method has been applied for the simulation of the complex dynamics of waves in excitable media. The discretization used either a classical finite volume scheme with explicit time discretization or Barkley's semi-implicit method with operator splitting. For both schemes, a detailed numerical study based on a series of simulation experiments showed that the automatic adaptation strategy is able to track the spatio-temporal pattern accurately at a substantially reduced computational cost. The nonlinear dynamics of complex multiscale patterns can thus be computed efficiently, also in the chaotic and turbulent regime which are currently beyond the frontiers of methods using regular discretizations.

In spite of modelling limitations associated with a simple system such as (1), the presented method could be of help in the control of more involved biological excitation waves thanks to the increased computational efficiency. We have also proposed the use of degenerate diffusion in the context of spiral waves simulation. The obtained results suggest such mechanism as an alternative way of numerically achieving sharper fronts in the simulation 
of spiral waves in excitable media. On the other hand, as the discussion of Examples 7 and 8 shows, it appears that degenerate diffusion leads to some intriguing phenomena (such as close-to-octagonal spirals) that seem to merit further in-depth investigation.

Insights gained from the study of these problems carried out via MR methods could help in reaching a better understanding of the processes involved in the spiral wave dynamics. Moreover, current extensions of this study to more general models are envisaged, including an anisotropic version of (1), and the modelling of the coupling of reaction-diffusion systems similar to (1), with a model of elasticity to consider motion of the excitable medium in the context of cardiac applications.

Acknowledgements RB acknowledges partial support by Fondecyt project 1090456, Fondap in Applied Mathematics, project 15000001, and BASAL project CMM, Universidad de Chile and Centro de Investigación en Ingeniería Matemática ( $\left.\mathrm{CI}^{2} \mathrm{MA}\right)$, Universidad de Concepción. RR acknowledges financial support by the European Research Council Advanced Grant "Mathcard, Mathematical Modelling and Simulation of the Cardiovascular System", project ERC-2008-AdG 227058. KS acknowledges financial support from the Agence Nationale de la Recherche, project "M2TFP". We acknowledge helpful comments made by two anonymous referees.

\section{References}

1. Aliev, R.R., Panfilov, A.V.: A simple two-variable model of cardiac excitation. Chaos Solitons Fractals 7, 293-301 (1996)

2. Barkley, D.: A model for fast computer simulation of waves in excitable media. Physica D 49, 61-70 (1991)

3. Barkley, D.: Spatial meandering. In: Kapral, R., Showalter, D. (eds.) Chemical Waves and Patterns, pp. 163-190. Kluwer, Dordrecht (1995)

4. Bendahmane, M., Bürger, R., Ruiz-Baier, R.: A multiresolution space-time adaptive scheme for the bidomain model in electrocardiology. Numer. Methods Partial Differ. Equ. (2010, to appear)

5. Bendahmane, M., Bürger, R., Ruiz-Baier, R., Schneider, K.: Adaptive multiresolution schemes with local time stepping for two-dimensional degenerate reaction-diffusion systems. Appl. Numer. Math. 59, 1668-1692 (2009)

6. Bendahmane, M., Bürger, R., Ruiz-Baier, R., Urbano, J.M.: On a doubly nonlinear diffusion model of chemotaxis with prevention of overcrowding. Math. Methods Appl. Sci. 32, 1704-1737 (2009)

7. Bihari, B.L., Harten, A.: Multiresolution schemes for the numerical solution of 2-D conservation laws I. SIAM J. Sci. Comput. 18, 315-354 (1997)

8. Bürger, R., Ruiz-Baier, R., Schneider, K., Sepúlveda, M.: Fully adaptive multiresolution schemes for strongly degenerate parabolic equations in one space dimension. M2AN Math. Model. Numer. Anal. 42, 535-563 (2008)

9. Carrillo, J.: Entropy solutions for nonlinear degenerate problems. Arch. Ration. Mech. Anal. 147, 269361 (1999)

10. Chen, X., Gao, C.: Well-posedness of a free boundary problem in the limit of slow-diffusion fast-reaction systems. J. Partial Differ. Equ. 19, 48-79 (2006)

11. Chiavassa, G., Donat, R.: Point value multiscale algorithms for 2D compressible flows. SIAM J. Sci. Comput. 23, 805-823 (2001)

12. Chiavassa, G., Donat, R., Müller, S.: Multiresolution-based adaptive schemes for hyperbolic conservation laws. In: Plewa, T., Linde, T., Weirs, V.G. (eds.) Adaptive Mesh Refinement-Theory and Applications, pp. 137-159. Springer, Berlin (2003)

13. Cohen, A., Kaber, S., Müller, S., Postel, M.: Fully adaptive multiresolution finite volume schemes for conservation laws. Math. Comput. 72, 183-225 (2003)

14. Cohen, A., Kaber, S., Postel, M.: Multiresolution analysis on triangles: application to conservation laws. In: Vielsmeier, R., Benkhaldoun, F., Hanel, D. (eds.) Finite Volumes for Complex Applications II. Hermès, Paris (1999)

15. Dahmen, W., Gottschlich-Müller, B., Müller, S.: Multiresolution schemes for conservation laws. Numer. Math. 88, 399-443 (2001)

16. Domingues, M., Gomes, S., Roussel, O., Schneider, K.: An adaptive multiresolution scheme with local time-stepping for evolutionary PDEs. J. Comput. Phys. 227, 3758-3780 (2008) 
17. Dowle, M., Mantel, R.M., Barkley, D.: Fast simulations of waves in three-dimensional excitable media. Int. J. Bifurc. Chaos 7, 2529-2545 (1997)

18. Eymard, R., Gallouët, T., Herbin, R.: Finite volume methods. In: Ciarlet, P.G., Lions, J.L. (eds.) Handbook of Numerical Analysis, vol. VII, pp. 713-1020. North-Holland, Amsterdam (2000)

19. Eymard, R., Gallouët, T., Herbin, R., Michel, A.: Convergence of a finite volume scheme for nonlinear degenerate parabolic equations. Numer. Math. 92, 41-82 (2002)

20. Fenton, F., Karma, A.: Vortex dynamics in 3D continuous myocardium with fiber rotation: filament instability and fibrillation. Chaos 8, 20-47 (1998)

21. Giga, Y., Goto, S., Ishii, H.: Global existence of weak solutions for interface equations coupled with diffusion equations. SIAM J. Math. Anal. 23, 821-835 (1992)

22. Goldstein, R.E.: Traveling-wave chemotaxis. Phys. Rev. Lett. 77, 775-778 (1996)

23. Goryachev, A., Kapral, R.: Spiral waves in chaotic systems. Phys. Rev. Lett. 76, 1619-1622 (1996)

24. Goryachev, A., Kapral, R.: Spiral waves in media with complex-excitable dynamics. Int. J. Bifurc. Chaos 11, 2243-2247 (1999)

25. Greenberg, J.M., Hastings, S.P.: Spatial patterns for discrete models of diffusion in excitable media. SIAM J. Appl. Math. 34, 515-523 (1978)

26. Harten, A.: Multiresolution algorithms for the numerical solution of hyperbolic conservation laws. Commun. Pure Appl. Math. 48, 1305-1342 (1995)

27. Heineken, W., Warnecke, G.: Partitioning methods for reaction-diffusion problems. Appl. Numer. Math. 56, 981-1000 (2006)

28. Herrero, M.A., Sastre, L.: Models of aggregation in Dictyostelium discoideum: on the track of spiral waves. Netw. Heterog. Media 1, 241-258 (2006)

29. Höfer, T., Sherratt, J.A., Maini, P.K.: Cellular pattern formation during Dictyostelium aggregation. Physica D 85, 425-444 (1995)

30. Holden, H., Karlsen, K.H., Risebro, N.H.: On uniqueness and existence of entropy solutions of weakly coupled systems of nonlinear degenerate parabolic equations. Electron. J. Differ. Equ. 2003, 1-31 (2003)

31. Hoyle, R.: Pattern Formation. Cambridge University Press, Cambridge (2006)

32. Hu, G., Xiao, J., Chua, L.O., Piska, L.: Controlling spiral waves in a model of two-dimensional arrays of Chua's circuits. Phys. Rev. Lett. 80, 1998 (1884-1887)

33. Jomaas, G., Bechtold, J.K., Lawa, C.K.: Spiral waves in expanding hydrogen-air flames: Experiment and theory. Proc. Combust. Inst. 31, 1039-1046 (2007)

34. Karlsen, K.H., Risebro, N.H.: Convergence of finite difference schemes for viscous and inviscid conservation laws with rough coefficients. M2AN Math. Model. Numer. Anal. 35, 239-269 (2001)

35. Keener, J.: Arrhythmias by dimension. In: Proc. Symp. Appl. Math. vol. 59, pp. 57-81. Am. Math. Soc., Providence (2002)

36. Keener, J., Sneyd, J.: Mathematical Physiology. I: Cellular Physiology, 2nd edn. Springer, New York (2009)

37. Keener, J., Sneyd, J.: Mathematical Physiology. II: Systems Physiology, 2nd edn. Springer, New York (2009)

38. Lee, K.J., Goldstein, R.E., Cox, E.C.: cAMP waves in Dictyostelium territories. Nonlinearity 15, C1-C5 (2002)

39. Margerit, D., Barkley, D.: Cookbook asymptotics for spiral and scroll waves in excitable media. Chaos 12, 636-649 (2002)

40. Mascia, C., Porretta, A., Terracina, A.: Non-homogeneous Dirichlet problems for degenerate parabolichyperbolic equations. Arch. Ration. Mech. Anal. 163, 87-124 (2002)

41. Michel, A., Vovelle, J.: Entropy formulation for parabolic degenerate equations with general Dirichlet boundary conditions and application to the convergence of FV methods. SIAM J. Numer. Anal. 41, 2262-2293 (2003)

42. Mikhailov, A.S., Showalter, K.: Control of waves patterns and turbulence in chemical systems. Phys. Rep. 425, 79-194 (2006)

43. Mitchell, A.: On the crest of a spiral wave. Nature 389, 547 (1997)

44. Müller, S.: Adaptive Multiscale Schemes for Conservation Laws. Springer, Berlin (2003)

45. Müller, S., Stiriba, Y.: Fully adaptive multiscale schemes for conservation laws employing locally varying time stepping. J. Sci. Comput. 30, 493-531 (2007)

46. Murray, J.D.: Mathematical Biology. II: Spatial Models and Biomedical Applications, 3rd edn. Springer, New York (2003)

47. Olmos, D., Shizgal, B.D.: Pseudospectral method of solution of the FitzHugh-Nagumo equation. Math. Comput. Simul. 79, 2258-2278 (2009)

48. Panfilov, V., Bayliss, A., Matkowsky, B.J.: Spiral flames. Appl. Math. Lett. 16, 131-135 (2003)

49. Pérez-Muñuzuri, A., Pérez-Muñuzuri, V., Pérez-Villar, V., Chua, L.O.: Spiral waves on a 2-D array of nonlinear circuits. IEEE Trans. Circuits Syst. 40, 872-877 (1993) 
50. Ramos, J.I.: Spiral wave break-up and planar front formation in two-dimensional reactive-diffusive media with straining. Appl. Math. Comput. 154, 697-711 (2004)

51. Roussel, O., Schneider, K., Tsigulin, A., Bockhorn, H.: A conservative fully adaptive multiresolution algorithm for parabolic PDEs. J. Comput. Phys. 188, 493-523 (2003)

52. Shajahan, T.K., Sinha, S., Pandit, R.: Spiral-wave dynamics depends sensitively on inhomogeneities in mathematical models of ventricular tissue. Phys. Rev. E 75, 011929 (2007)

53. Shardlow, T.: Numerical simulation of stochastic PDEs for excitable media. J. Comput. Appl. Math. 175, 429-446 (2005)

54. Scheel, A.: Bifurcation to spiral waves in reaction-diffusion systems. SIAM J. Math. Anal. 29, 13991418 (1998)

55. Smith, G.D.: Numerical Solution of Partial Differential Equations: Finite Difference Methods. Clarendon Press, Oxford (1985)

56. Tomchik, K.J., Devreotes, P.N.: Adenosine $3^{\prime}, 5^{\prime}$-monophosphate waves in Dictyostelium discoideum: a demonstration by isotope dilution-fluorography. Science 212, 443-446 (1981)

57. Tung, L.: Electroporation of cardiac cells. In: Nickoloff, J.A. (ed.) Animal Cell Electroporation and Electrofusion Protocols. Methods in Molecular Biology, vol. 48, pp. 253-271 (1995)

58. Tyson, J.J., Murray, J.D.: Cyclic AMP waves during aggregation of Dictyostelium amoebae. Development 106, 421-426 (1989)

59. Rauch, J.: Global existence for the FitzHugh-Nagumo equations. Commun. Partial Differ. Equ. 1, 609621 (1976)

60. van Oss, C., Panfilov, A.V., Hogeweg, P., Siegert, F., Weijer, C.J.: Spatial pattern formation during aggregation of the slime mold Dictyostelium discoideum. J. Theor. Biol. 181, 203-213 (1996)

61. Vanag, V.K., Epstein, I.R.: Segmented spiral waves in a reaction-diffusion system. Proc. Natl. Acad. Sci. 100, 14635-14638 (2003)

62. Weiser, M.: Pointwise nonlinear scaling for reaction-diffusion equations. Appl. Numer. Math. 59, 2009 (1858-1869)

63. Winfree, A.T.: The prehistory of the Belousov-Zhabotinsky oscillator. J. Chem. Educ. 61, 661-663 (1984)

64. Witelski, T.P.: Segregation and mixing in degenerate diffusion in population dynamics. J. Math. Biol. 35, 695-712 (1997)

65. Xu, W., Hu, D., Lei, A., Li, Q.S.: Single spiral wave induced by noise. Appl. Math. Comput. 171, 703709 (2005)

66. Zaikin, A.N., Zhabotinsky, A.M.: Concentration wave propagation in two-dimensional liquid-phase selfoscillating system. Nature 225, 535-537 (1970)

67. Zhabotinsky, A.M.: A history of chemical oscillations and waves. Chaos 1, 379-386 (1991)

68. Zhang, H., Hu, B., Hu, G.: Suppression of spiral waves and spatiotemporal chaos by generating target waves in excitable media. Phys. Rev. E 68, 026134 (2003) 